\title{
TURBULENCE, REPRESENTATIONS, AND TRACE-PRESERVING ACTIONS
}

\author{
DAVID KERR, HANFENG LI, AND MIKAËL PICHOT
}

\begin{abstract}
We establish criteria for turbulence in certain spaces of $C^{*}$-algebra representations and apply this to the problem of nonclassifiability by countable structures for group actions on a standard atomless probability space $(X, \mu)$ and on the hyperfinite $\mathrm{II}_{1}$ factor $R$. We also prove that the conjugacy action on the space of free actions of a countably infinite amenable group on $R$ is turbulent, and that the conjugacy action on the space of ergodic measure-preserving flows on $(X, \mu)$ is generically turbulent.
\end{abstract}

\section{INTRODUCTION}

Descriptive set theory provides a natural framework for the study of the complexity of classification problems in analysis and dynamics [31, 26]. Often one has a collection of objects that can be viewed as elements in a Polish space $X$ and an equivalence relation $E$ on $X$ encoding the isomorphism relation between the objects. Consider for example the set of unitary operators on a separable Hilbert space with the strong operator topology or the set of measure-preserving transformations of a standard probability space with the weak topology, each under the relation of conjugacy. We may then attempt to gauge the complexity of $E$ by the way it relates descriptively to other equivalence relations. Given another equivalence relation $F$ on a standard Borel space $Y$, one says that $E$ is Borel reducible to $F$ if there is a Borel map $\theta: X \rightarrow Y$ such that, for all $x_{1}, x_{2} \in X, x_{1} E x_{2}$ if and only if $\theta\left(x_{1}\right) F \theta\left(x_{2}\right)$. If we can Borel reduce $E$ to a relation on objects which are in some sense better understood, we may reasonably claim to have a classification theory.

The relation $E$ is said to be smooth if it can be Borel reduced to equality on $\mathbb{R}$, i.e., if we can assign numerical invariants in a Borel manner. By a theorem of Glimm, the space of irreducible representations of a separable $C^{*}$-algebra $A$ is smooth precisely when $A$ is type I (see Section 6.8 of [41]). The theorem of Ornstein asserting that entropy is a complete invariant for Bernoulli shifts provides another example of smoothness [39]. To show that a Borel equivalence relation $E$ on $X$ is not smooth, it suffices to demonstrate the existence of a Borel probability measure on $X$ which is ergodic (i.e., every invariant Borel set has measure 0 or 1 ) and is zero on every equivalence class. The relation $E_{0}$ of tail equivalence on $\{0,1\}^{\mathbb{N}}$ satisfies this proper ergodicity condition in a prototypical way, and indeed when $E$ is Borel the continuous embeddability of $E_{0}$ into $E$ is a universal obstruction to smoothness [24]. There is also a topological version of the proper ergodicity obstruction to smoothness via Baire category in the case that $E$ arises as the orbit equivalence relation of the continuous action of a Polish group on $X$, namely that every equivalence class is both dense and meager (see Section 3.1 of [26]).

Date: August 11, 2008. 
At a higher level of complexity is the notion of classification by countable structures, which means that $E$ can be Borel reduced to the isomorphism relation on the space of countable structures of some countable language as implemented by the logic action of the infinite permutation group $S_{\infty}$ with its unique Polish topology [26, Defn. 2.38]. Equivalently, $E$ can be Borel reduced to the orbit equivalence relation of a Borel action of $S_{\infty}$ on a Polish space [2, Sect. 2.7]. Non-smooth examples of this are the Halmos-von Neumann classification of discrete spectrum transformations by their sets of eigenvalues 23. and the Giordano-Putnam-Skau classification of minimal homeomorphisms of the Cantor set up to strong orbit equivalence by countable ordered Abelian groups [18]. Note that the isomorphism relation on any type of countable algebraic structure can be encoded as a continuous $S_{\infty}$-action on a Polish space (see Example 2 in [17]).

In analogy with the topological proper ergodicity obstruction to smoothness, Hjorth developed the notion of turbulence as a means for demonstrating nonclassifiability by countable structures [26]. Let $G$ be a Polish group acting continuously on a Polish space $X$. For an $x \in X$ and open sets $U \subseteq X$ and $V \subseteq G$ containing $x$ and $e$, respectively, we define the local orbit $\mathcal{O}(x, U, V)$ as the set of all $y \in U$ for which there are $g_{1}, g_{2}, \ldots, g_{n} \in V$ such that $g_{k} g_{k-1} \cdots g_{1} x \in U$ for each $k=1, \ldots, n-1$ and $g_{n} g_{n-1} \cdots g_{1} x=y$. A point $x \in X$ is turbulent if for all nonempty open sets $U \subseteq X$ and $V \subseteq G$ containing $x$ and $e$, respectively, the closure of $\mathcal{O}(x, U, V)$ has nonempty interior. The action is turbulent if every orbit is dense and meager and every point is turbulent. Section 3.2 of [26] shows that if the action of $G$ on $X$ is turbulent then whenever $F$ is an equivalence relation arising from a continuous action of $S_{\infty}$ on a Polish space $Y$ and $\theta: X \rightarrow Y$ is a Baire measurable function such that $x_{1} E x_{2}$ implies $\theta\left(x_{1}\right) F \theta\left(x_{2}\right)$, there exists a comeager set $C \subseteq X$ such that $\theta\left(x_{1}\right) F \theta\left(x_{2}\right)$ for all $x_{1}, x_{2} \in C$. As a consequence the orbit equivalence relation on $X$ does not admit classification by countable structures. In fact to obtain this conclusion it suffices to show that the action is generically turbulent, which can be expressed by saying that some orbit is dense, every orbit is meager, and some point is turbulent (see Definiton 3.20 and Theorem 3.21 in [26]). By Theorem 3.21 of [26], if the action of $G$ on $X$ is generically turbulent then there is a $G$-invariant dense $G_{\delta}$ subset of $X$ on which the action is turbulent.

Turbulence has now been established in several situations. Hjorth showed in [25] that if $G$ is a countably infinite group which is not a finite extension of an Abelian group (which in this case is equivalent to $G$ not being type I by a result of Thoma) then the space of irreducible representations of $G$ on a separable infinite-dimensional Hilbert space $\mathcal{H}$ under the conjugation action of the unitary group $\mathcal{U}(\mathcal{H})$ admits an invariant $G_{\delta}$ subset on which the action is turbulent. Hjorth's argument yields the same conclusion more generally for the space of irreducible representations of any separable non-type I $C^{*}$-algebra on $\mathcal{H}$. Within the type I realm, Kechris and Sofronidis established generic turbulence for the conjugation actions of $\mathcal{U}(\mathcal{H})$ on itself and on the space of self-adjoint operators of norm at most one with the strong topology [32].

Suppose now that $G$ is a countably infinite group and consider the Polish space $\operatorname{Act}(G, X, \mu)$ of actions of $G$ by measure-preserving transformations on a standard atomless probability space $(X, \mu)$ under the weak topology, with the conjugation action of $\operatorname{Aut}(X, \mu)=\operatorname{Act}(\mathbb{Z}, X, \mu)$. Hjorth constructed a turbulent action which Borel reduces to the conjugacy relation on the space of ergodic automorphisms in $\operatorname{Aut}(G, X, \mu)$ [27] 
and used turbulence in spaces of irreducible representations to show nonclassifiability by countable structures for the subspace of free weakly mixing actions when $G$ is not a finite extension of an Abelian group (see Theorem 13.7 in [31]). Foreman and Weiss proved that the action of $\operatorname{Aut}(X, \mu)$ on the space of free ergodic actions in $\operatorname{Act}(G, X, \mu)$ is turbulent when $G$ is amenable, using entropy and disjointness to obtain the meagerness of orbits and the Rokhlin lemma and orbit equivalence to verify that every point is turbulent [17. Free weakly mixing actions of any countably infinite $G$ considered up to unitary conjugacy also do not admit classification by countable structures [31, Thm. 13.8].

One of the main goals of the present paper is to develop a general spectral approach to the identification of turbulent behaviour in spaces of representations and actions. We prove that, for a separable $C^{*}$-algebra $A$ and a separable infinite-dimensional Hilbert space $\mathcal{H}$, the action of $\mathcal{U}(\mathcal{H})$ on the Polish space of faithful essential nondegenerate representations of $A$ on $\mathcal{H}$ has the property that every point is turbulent and has dense orbit, while the meagerness of all orbits is equivalent to the isolated points of the spectrum $\hat{A}$ not being dense, so that the action is turbulent precisely in this case. The action of $\mathcal{U}(\mathcal{H})$ on the Polish space of all nondegenerate representations of $A$ on $\mathcal{H}$ is turbulent precisely when $A$ is simple and not isomorphic to the compact operators on some Hilbert space. Furthermore, the orbit equivalence relation on the space of nondegenerate representations does not admit classification by countable structures as soon as $\hat{A}$ is uncountable (if $\hat{A}$ is countable then the classification of nondegenerate representations on $\mathcal{H}$ is a matter of counting multiplicities of irreducible subrepresentations and hence is smooth).

This spectral picture leads in particular to a unified proof of nonclassifiability by countable structures for free weakly mixing actions of countably infinite $G$ that does not rely on the type I/non-type I dichotomy, and also allows us to extend the conclusion to weakly mixing actions of many nondiscrete $G$ of type $\mathrm{I}$, such as $\mathbb{R}^{d}$ and $\operatorname{SL}(2, \mathbb{R})$. We show moreover that the same nonclassifiability statements hold if we replace $(X, \mu)$ with the hyperfinite $\mathrm{II}_{1}$ factor $R$. What is of particular interest about the noncommutative context is the fact that, under amenability assumptions, actions on a factor can be classified up to cocycle conjugacy by cohomological invariants. For actions of countable amenable groups on $R$ this was done by Ocneanu [38, Thm. 2.6], extending the fundamental work of Connes on single automorphisms [9]. For finite groups one can go further and produce a classification up to conjugacy, as was done by Connes in the periodic case [10] and Jones in general [28].

In the case that the acting group $G$ is countably infinite and amenable, we prove that the action of the automorphism group of $R$ on the space of free $G$-actions on $R$ is turbulent. To obtain the meagerness of orbits we follow the idea of Foreman and Weiss of using entropy and disjointness, although in the noncommutative situation a different technical perspective is required. We show for general second countable locally compact $G$ that there exists a turbulent point with dense orbit, and deduce from this that every point is turbulent when $G$ is countably infinite and amenable by applying Ocneanu's result that any two free actions are cocycle conjugate in this case, with bounds on the cocycle [38, Thm. 1.4]. Our method for demonstrating the existence of a turbulent point with dense orbit also works in the commutative situation, yielding a proof that works equally well for nondiscrete $G$ and does not involve orbit equivalence (compare [17] and Section 5 in 
[31]). Using this we deduce that the action of $\operatorname{Aut}(X, \mu)$ on the space of ergodic measurepreserving flows on $(X, \mu)$ is generically turbulent.

The paper contains five sections beyond the introduction. Section 2 contains results on turbulence in spaces of $C^{*}$-algebra representations, while Section 3 discusses the ramifications of these for group representations. In Section 4 we discuss freeness and weak mixing and establish our nonclassifiability results for actions based on the spectral analysis of Section 2. Section 5 contains the proof of turbulence in the space of free actions of a countably infinite amenable group on $R$. Finally, in Section 6 we show generic turbulence in the space of ergodic measure-preserving flows.

Acknowledgements. D.K. was partially supported by NSF grant DMS-0600907. H.L. was partially supported by NSF grant DMS-0701414. M.P. was supported by the EPDI and a JSPS fellowship for European researchers. M.P. is grateful to the Max-Planck Institut für Mathematik for hospitality and to Yasuyuki Kawahigashi for hosting his stay at the University of Tokyo. The initial stages of this work were carried out during a visit of M.P. to Texas A\&M University in July 2007.

\section{Representations of $C^{*}$-Algebras}

Let $A$ be a separable $C^{*}$-algebra and $\mathcal{H}$ a separable infinite-dimensional Hilbert space. A representation $\pi: A \rightarrow \mathcal{B}(\mathcal{H})$ is said to be essential if $\pi(A) \cap \mathcal{K}(\mathcal{H})=\{0\}$, where $\mathcal{K}(\mathcal{H})$ denotes the $C^{*}$-algebra of compact operators on $\mathcal{H}$. We say that $\pi$ is nondegenerate if for every nonzero vector $\xi \in \mathcal{H}$ there is an $a \in A$ such that $\pi(a) \xi \neq 0$. If $\left\{h_{\eta}\right\}_{\eta}$ is an approximate unit for $A$, then $\pi$ is nondegenerate if and only if $\pi\left(h_{\eta}\right)$ tends to the identity operator on $\mathcal{H}$ in the strong operator topology. In particular, if $A$ is unital then $\pi$ is nondegenerate if and only if it is unital. By Voiculescu's theorem, any two faithful essential nondegenerate representations $\pi_{1}$ and $\pi_{2}$ of $A$ on separable infinite-dimensional Hilbert spaces $\mathcal{H}_{1}$ and $\mathcal{H}_{2}$, respectively, are approximately unitarily equivalent in the sense that there exists a sequence of unitary operators $U_{n}: \mathcal{H}_{1} \rightarrow \mathcal{H}_{2}$ such that

$$
\lim _{n \rightarrow \infty}\left\|U_{n} \pi_{1}(a) U_{n}^{-1}-\pi_{2}(a)\right\|=0
$$

for all $a \in A$, and every representation of $A$ is approximately unitarily equivalent to a direct sum of irreducible representations [48]. (see also [13, 7]).

We write $\operatorname{Rep}(A, \mathcal{H})$ for the Polish space of all nondegenerate representations of $A$ on $\mathcal{H}$ whose topology has as a basis the sets

$$
Y_{\pi, F, \Omega, \varepsilon}=\{\rho \in \operatorname{Rep}(A, \mathcal{H}):|\langle(\rho(a)-\pi(a)) \xi, \zeta\rangle|<\varepsilon \text { for all } a \in F \text { and } \xi, \zeta \in \Omega\}
$$

where $\pi \in \operatorname{Rep}(G, \mathcal{H}), F$ is a finite subset of $A, \Omega$ is a finite subset of $\mathcal{H}$, and $\varepsilon>0$. Sets of the form

$$
\{\rho \in \operatorname{Rep}(A, \mathcal{H}):\|(\rho(a)-\pi(a)) \xi\|<\varepsilon \text { for all } a \in F \text { and } \xi \in \Omega\}
$$

with the same type of $\pi, F, \Omega$, and $\varepsilon$ also form a basis for the topology. We write $\mathcal{U}(\mathcal{H})$ for the group of unitary operators on $\mathcal{H}$ equipped with the relative strong operator (equivalently, relative weak operator) topology, under which it is a Polish group. We will be concerned with the continuous action $(U, \pi) \mapsto \operatorname{Ad} U \circ \pi$ of $\mathcal{U}(\mathcal{H})$ on $\operatorname{Rep}(A, \mathcal{H})$.

The spectrum $\hat{A}$ of $A$ is defined as the set of unitary equivalence classes of irreducible representations of $A$ equipped with the topology under which the canonical map from $\hat{A}$ 
onto the primitive ideal space of $A$ with the Jacobson topology is open and continuous [41, Sect. 4.1]. As usual we identify elements in $\hat{A}$ with their representatives. As we are assuming $A$ to be separable, the topology on $\hat{A}$ is second countable [15, Prop. 3.3.4]. This topology can also be described in terms of weak containment or, in the case that $A$ has no finite-dimensional irreducible representations, as the quotient topology on unitary equivalence classes of irreducible representations in $\operatorname{Rep}(A, \mathcal{H})$ (if $A$ has finite-dimensional irreducible representations then one can stabilize and use the canonical homeomorphism from $\widehat{A \otimes \mathcal{K}}$ to $\hat{A}$ ) [15, Sects. 3.4 and 3.5][5, II.6.5.16].

In the proof of the following lemma we use a rotation trick as in 25. For a closed linear subspace $E$ of $\mathcal{H}$ we write $P_{E}$ for the orthogonal projection of $\mathcal{H}$ onto $E$.

Lemma 2.1. Let $\pi \in \operatorname{Rep}(A, \mathcal{H})$. If $\pi$ is faithful and essential then it has dense orbit and is a turbulent point for the action of $\mathcal{U}(\mathcal{H})$. If $\pi$ is not faithful or not essential then its orbit is nowhere dense.

Proof. Suppose first that $\pi$ is faithful and essential. To establish turbulence, let $Y$ be a neighbourhood of $\pi$ in $\operatorname{Rep}(A, \mathcal{H})$ and $Z$ a neighbourhood of 1 in $\mathcal{U}(\mathcal{H})$. We will show that the closure of the local orbit $\mathcal{O}(\pi, Y, Z)$ has nonempty interior. We may suppose by shrinking $Y$ and $Z$ if necessary that $Y=Y_{\pi, K, \Omega, \varepsilon}$ and $Z=Z_{1, \Omega, \varepsilon}$ where $K$ is a finite subset of $A, \Omega$ is a finite subset of the unit ball of $\mathcal{H}$, and $\varepsilon>0$. Suppose that we are given a $\sigma \in Y$. Let $L$ be a finite subset of $A, \Upsilon$ a finite subset of the unit ball of $\mathcal{H}$, and $\delta>0$. We will construct a norm continuous path of unitaries $W_{t} \in \mathcal{U}(\mathcal{H})$ for $t \in[0, \pi / 2]$ such that $W_{0}=\mathbf{1}$, Ad $W_{t} \circ \pi \in Y$ for every $t \in[0, \pi / 2]$, and $\operatorname{Ad} W_{\pi / 2} \circ \pi \in Y_{\sigma, L, \Upsilon, \delta}$. This will show that $\sigma$ lies in the closure of $\mathcal{O}(\pi, Y, Z)$, since the continuity of the path permits us to find $t_{0}=0<t_{1}<t_{2}<\cdots<t_{m}=\pi / 2$ such that $W_{t_{i}} W_{t_{i-1}}^{-1} \in Z$ for each $i=1, \ldots, m$.

We may assume that $\Upsilon$ contains $\Omega$ and that $\delta$ is small enough so that $\|\sigma(a) \xi-\pi(a) \xi\|+$ $\delta<\varepsilon$ for all $a \in K$ and $\xi \in \Omega$. Let $E$ be the subspace spanned by $\Upsilon \cup \pi(K) \Upsilon \cup \sigma(L) \Upsilon$. Since $\pi$ is faithful and essential, by the matrix version of Glimm's lemma [8] (see also Lemma II.5.2 in [13] and the paragraph following it) there is an isometry $V: E \rightarrow \mathcal{H}$ such that $V E \perp \operatorname{span}\left(E \cup \pi(K \cup L)^{*} E\right)$ and $\left\|V^{*} \pi(a) V-\left.P_{E} \sigma(a)\right|_{E}\right\|<\delta$ for all $a \in K \cup L$. For each $t \in[0, \pi / 2]$ let $W_{t}$ be the unitary operator on $\mathcal{H}$ which is the identity on $(E \oplus V E)^{\perp}$ and acts on $E \oplus V E$ in $2 \times 2$ block form as

$$
\left[\begin{array}{cc}
\cos (t) \mathbf{1} & \sin (t) V^{*} \\
-\sin (t) V & \cos (t) \mathbf{1}
\end{array}\right]
$$

Let $t \in[0, \pi / 2], a \in K \cup L$, and $\xi \in \Upsilon$. Since $\left\|P_{V E} \pi(a) V \xi-V \sigma(a) \xi\right\|<\delta$ and $\pi(a) V \xi \in E^{\perp}$ we have, writing $c=\cos t$ and $s=\sin t$,

$$
\begin{aligned}
W_{t} \pi(a) W_{t}^{-1} \xi & =W_{t}(c \pi(a) \xi+s \pi(a) V \xi) \\
& \approx_{\delta} W_{t}\left(c \pi(a) \xi+s V \sigma(a) \xi+s P_{E \oplus V E}^{\perp} \pi(a) V \xi\right) \\
& =c^{2} \pi(a) \xi+s^{2} \sigma(a) \xi+c s V(-\pi(a) \xi+\sigma(a) \xi)+s P_{E \oplus V E}^{\perp} \pi(a) V \xi .
\end{aligned}
$$

It follows that if $a \in K$ and $\xi \in \Omega$ then

$$
\begin{aligned}
\left\|P_{E}\left(W_{t} \pi(a) W_{t}^{-1} \xi-\pi(a) \xi\right)\right\| & \leq\left\|c^{2} \pi(a) \xi+s^{2} \sigma(a) \xi-\pi(a) \xi\right\|+\delta \\
& =s^{2}\|\sigma(a) \xi-\pi(a) \xi\|+\delta
\end{aligned}
$$


$<\varepsilon$.

and so $\operatorname{Ad} W_{t} \circ \pi \in Y$ since $\Omega$ is contained in the unit ball of $E$. In the case $t=\pi / 2$ and $a \in L$ we obtain $\left\|P_{E}\left(W_{\pi / 2} \pi(a) W_{\pi / 2}^{-1} \xi-\sigma(a) \xi\right)\right\|<\delta$ and so $\operatorname{Ad} W_{\pi / 2} \circ \pi \in Y_{\sigma, L, \Upsilon, \delta}$ since $\Upsilon$ is contained in the unit ball of $E$. We thus conclude that $\sigma$ lies in the closure of $\mathcal{O}(\pi, Y, Z)$ and hence that $\pi$ is a turbulent point for the action of $\mathcal{U}(\mathcal{H})$. Moreover, since we have shown that $Y \subseteq \overline{\mathcal{O}(\pi, Y, Z)}$ for any $Y$ of the form $Y_{\pi, K, \Omega, \varepsilon}$, we see that the orbit of $\sigma$ is dense.

Now if $\pi$ is any faithful representation in $\operatorname{Rep}(A, \mathcal{H})$ then for every $a \in A$ we evidently have $\sigma(a) \neq 0$ for all $\sigma$ in some neighbourhood of $\pi$. Since the faithful representations are dense in $\operatorname{Rep}(A, \mathcal{H})$ by the first paragraph, we deduce that the orbit of every nonfaithful representation is nowhere dense.

Suppose finally that $\pi$ is a representation in $\operatorname{Rep}(A, \mathcal{H})$ which is not essential. Then $\pi(A)$ contains a nonzero positive compact operator, and hence by the functional calculus there is an $a \in A$ such that $\pi(a)$ is nonzero and of finite rank. Take a faithful essential representation $\sigma \in \operatorname{Rep}\left(G, \mathcal{H}\right.$ ) (for example, a representation unitarily equivalent to $\rho^{\oplus \mathbb{N}}$ for any faithful $\rho \in \operatorname{Rep}(G, \mathcal{H})$ ). It is readily seen that for every $\sigma^{\prime}$ in some neighbourhood of $\sigma$ the range of $\sigma^{\prime}(a)$ has dimension larger than the rank of $\pi(a)$. Since the orbit of $\sigma$ is dense as shown above, we conclude that the orbit of $\pi$ is nowhere dense.

Since for an action of a group on a second countable topological space the set of points with dense orbit is a $G_{\delta}$, we obtain from Lemma 2.1 the following.

Lemma 2.2. The set of faithful essential representations in $\operatorname{Rep}(A, \mathcal{H})$ is a dense $G_{\delta}$.

The following was observed for unitary representations of countable groups in Proposition H.2 of [31] but the same argument applies more generally.

Lemma 2.3. Let $\pi, \sigma \in \operatorname{Rep}(A, \mathcal{H})$ and let $\rho$ be an element of $\operatorname{Rep}(A, \mathcal{H})$ unitarily conjugate to $\sigma^{\oplus \mathbb{N}}$. Then $\pi$ is weakly contained in $\sigma$ if and only if $\pi$ lies in the orbit closure of $\rho$.

For each open set $U \subseteq \hat{A}$ there is a closed ideal $I \subseteq A$ such that $U$ is equal to the set $\hat{A}^{I}$ of all $\sigma \in \hat{A}$ for which $\sigma(I) \neq 0$, and the restriction map $\hat{A}^{I} \rightarrow \hat{I}$ is a homeomorphism [15. Sect. 3.2]. This sets up a bijective correspondence between the open subsets of $\hat{A}$ and the closed ideals of $A$, and for each closed ideal $I$ we regard $\hat{I}$ as an open subset of $\hat{A}$.

We write $\hat{A}_{\mathrm{i}}$ for the set of isolated points in $\hat{A}$. This set is countable because $\hat{A}$ is second countable, which follows from the separability of $A$ [15, Prop. 3.3.4].

Lemma 2.4. Let $A$ be a separable $C^{*}$-algebra such that $\hat{A}_{\mathrm{i}}$ is not dense in $\hat{A}$. Let I be the closed ideal of $A$ for which $\hat{I}$ is the complement of the closure of $\hat{A}_{\mathrm{i}}$. Let $\pi \in \operatorname{Rep}(A, \mathcal{H})$ and set $\mathcal{H}_{I}=\overline{\pi(I) \mathcal{H}}$. Then the set $\left(\left.\pi\right|_{\mathcal{H}_{I}}\right)^{\perp}$ of representations in $\operatorname{Rep}(A, \mathcal{H})$ which are disjoint from $\left.\pi\right|_{\mathcal{H}_{I}}$ is a dense $G_{\delta}$.

Proof. By Lemma 3.7.3 of [15], $\left(\left.\pi\right|_{\mathcal{H}_{I}}\right)^{\perp}$ is a $G_{\delta}$ subset of $\operatorname{Rep}(A, \mathcal{H})$. By Voiculescu's theorem [48, Cor. 1.6], the orbit closure of a given element of $\operatorname{Rep}(A, \mathcal{H})$ is the same as the orbit closure of some $\pi^{\prime}=\pi_{0} \oplus \bigoplus_{k \in K} \pi_{k}$ with $\pi_{0}(I)=\{0\}, K$ a countable index set, and $\pi_{k} \in \hat{I}$ for each $k \in K$. To show that $\left(\left.\pi\right|_{\mathcal{H}_{I}}\right)^{\perp}$ is dense, it suffices to show that $\pi^{\prime}$ is in the orbit closure of some element in $\left(\left.\pi\right|_{\mathcal{H}_{I}}\right)^{\perp}$. 
We first argue that each nonempty open subset of $\hat{I}$ is uncountable. Indeed suppose to the contrary that there is a countable open set $U \subseteq \hat{I}$. Let $I_{0}$ be the closed ideal of $A$ such that $\hat{I}_{0}=U$. By Lemma 1.3 of [49], $\hat{J}$ has an isolated point. This point is also isolated when viewed as an element of $\hat{A}$, yielding a contradiction.

Since $\mathcal{H}_{I}$ is separable, the set $D$ of irreducible subrepresentations of $\left.\pi\right|_{\mathcal{H}_{I}}$ is countable. Thus, since $\hat{I}$ is second countable and each of its nonempty open subsets is uncountable, we can construct a countable set $E \subseteq \hat{I} \backslash D$ which contains $\pi_{k}$ in its closure for each $k \in K$. Then $\left.\pi\right|_{\mathcal{H}_{I}}$ is disjoint from $\pi_{0} \oplus \bigoplus_{\rho \in E} \rho^{\oplus \mathbb{N}}$, and $\pi^{\prime}$ is weakly contained in $\pi_{0} \oplus \bigoplus_{\rho \in E} \rho^{\oplus \mathbb{N}}$ [15. Thm. 3.4.10] and hence lies in the orbit closure of every representation in $\operatorname{Rep}(A, \mathcal{H})$ unitarily conjugate to $\pi_{0} \oplus \bigoplus_{\rho \in E} \rho^{\oplus \mathbb{N}}$ by Lemma 2.3. This finishes the proof.

Write $\operatorname{FE}(A, \mathcal{H})$ for the $\mathcal{U}(\mathcal{H})$-invariant set of faithful essential representations in $\operatorname{Rep}(A, \mathcal{H})$, which is a dense $G_{\delta}$ by Lemma 2.2 .

Theorem 2.5. Let $A$ be a separable $C^{*}$-algebra. If $\hat{A}_{\mathrm{i}}$ is dense in $\hat{A}$ then the action of $\mathcal{U}(\mathcal{H})$ on $\operatorname{Rep}(A, \mathcal{H})$ has a dense $G_{\delta}$ orbit, while if $\hat{A}_{\mathrm{i}}$ is not dense in $\hat{A}$ then the restriction of the action to $\operatorname{FE}(A, \mathcal{H})$ is turbulent. Furthermore, the action on $\operatorname{Rep}(A, \mathcal{H})$ is turbulent precisely when $A$ is simple and not isomorphic to the compact operators on some Hilbert space.

Proof. Suppose first that $\hat{A}_{\mathrm{i}}$ is dense in $\hat{A}$. Let $I$ be the closed ideal of $A$ such that $\hat{I}=\hat{A}_{\mathrm{i}}$. For each $\pi \in \hat{A}_{\mathrm{i}}$ let $I_{\pi}$ be the closed ideal of $A$ such that $\hat{I}_{\pi}=\{\pi\}$. Note that the set $V$ of all representations in $\operatorname{Rep}(A, \mathcal{H})$ which are nondegenerate on $I$ can be expressed as

$$
\bigcap_{n=1}^{\infty}\left\{\sigma \in \operatorname{Rep}(G, \mathcal{H}):\left\|\sigma(a) \xi_{n}\right\|>\frac{1}{2}\left\|\xi_{n}\right\| \text { for some } a \text { in the unit ball of } A\right\}
$$

for a given dense sequence $\left\{\xi_{n}\right\}_{n=1}^{\infty}$ in the unit sphere of $\mathcal{H}$ and hence is a $G_{\delta}$. Given a $\pi \in \hat{I}$, choose a projection $p_{\pi} \in I_{\pi}$ such that $\pi\left(p_{\pi}\right)$ has rank one. Then for every $n \in \mathbb{N}$ the set $V_{\pi, n}$ of all $\pi \in \operatorname{Rep}(A, \mathcal{H})$ such that $\operatorname{Tr}\left(\sigma\left(p_{\pi}\right)\right)>n$ is easily seen to be open by expressing Tr in terms of a fixed orthonormal basis of $\mathcal{H}$. As $\hat{A}$ is second countable, $\hat{I}$ is countable. Set $\rho=\bigoplus_{\pi \in \hat{A}_{\mathrm{i}}} \pi^{\oplus \mathbb{N}}$. By Lemma 1.4 of [49], every representation of $I$ is a direct sum of irreducible representations. Consequently the orbit of $\rho$ is precisely $V \cap \bigcap_{\pi \in \hat{A}_{\mathrm{i}}} \bigcap_{n \in \mathbb{N}} V_{\pi, n}$, a $G_{\delta}$ subset of $\operatorname{Rep}(A, \mathcal{H})$. Moreover, since by Voiculescu's theorem the orbit closure of any element in $\operatorname{Rep}(A, \mathcal{H})$ is equal to the orbit closure of some direct sum of irreducible representations [48, Cor. 1.6], we see by [15, Thm. 3.4.10] and Lemma 2.3 that the orbit of $\pi$ is dense in $\operatorname{Rep}(A, \mathcal{H})$.

Suppose now that $\hat{A}_{\mathrm{i}}$ is not dense in $\hat{A}$. To establish that the action of $\mathcal{U}(\mathcal{H})$ on $\operatorname{FE}(A, \mathcal{H})$ is turbulent, it suffices by Lemma 2.1 to show that every orbit in $\operatorname{FE}(A, \mathcal{H})$ is meager. Let $\pi \in \operatorname{FE}(A, \mathcal{H})$. Let $I$ be closed ideal of $A$ such that $\hat{I}$ is the complement of the closure of $\hat{A}_{\mathrm{i}}$ in $\hat{A}$. Then $\pi(I) \neq\{0\}$ by faithfulness, and so the orbit of $\pi$ is meager by Lemma 2.4. Thus every orbit in $\operatorname{FE}(A, \mathcal{H})$ is meager and we have turbulence.

Finally, if $A$ is simple and not isomorphic to the compact operators on some Hilbert space then every representation is faithful and essential and the topology on $\hat{A}$ is trivial. Thus by Lemma 2.1 every orbit in $\operatorname{Rep}(A, \mathcal{H})$ is dense and so the action is turbulent in view of what we know from above. If $A$ is isomorphic to the compact operators on some 
Hilbert space then its spectrum is a singleton and so from above there is a dense $G_{\delta}$ orbit in $\operatorname{Rep}(A, \mathcal{H})$. If $A$ is not simple then it has a nontrivial quotient and hence a nonfaithful representation in $\operatorname{Rep}(A, \mathcal{H})$, and the orbit of this representation is nowhere dense by Lemma 2.1, so that the action fails to be turbulent.

Corollary 2.6. Let $A$ be a separable unital antiliminary $C^{*}$-algebra. Then the action of $\mathcal{U}(\mathcal{H})$ on $\operatorname{Rep}(A, \mathcal{H})$ is generically turbulent.

Proof. This follows from the theorem because the existence of an isolated point of $\hat{A}$ would yield an ideal isomorphic to the compact operators on a separable Hilbert space [49, Lemma 1.3], contradicting antiliminarity.

If $A$ is a separable $C^{*}$-algebra such that $\hat{A}$ is countable, then every representation of $\operatorname{Rep}(A, \mathcal{H})$ is a direct sum of irreducible representations [49, Lemma 1.4], and the associated multiplicity function $\hat{A} \rightarrow\{0,1, \ldots, \infty\}$ is a complete invariant for unitary equivalence. So in this case the classification of elements in $\operatorname{Rep}(A, \mathcal{H})$ up to unitary equivalence is smooth. On the other hand:

Theorem 2.7. Let $A$ be a separable $C^{*}$-algebra such that $\hat{A}$ is uncountable. Then the elements of $\operatorname{Rep}(A, \mathcal{H})$ up to unitary equivalence do not admit classification by countable structures.

Proof. By the Cantor-Bendixson theorem, the set $P$ of condensation points in $\hat{A}$ is perfect (and in particular closed) and its complement is countable. Since $\hat{A}$ is uncountable, $P$ is nonempty. Thus there is an ideal $I \subseteq A$ such that $P$ is equal to the set $\hat{A}_{I}$ of all $\sigma \in \hat{A}$ for which $\sigma(I)=0$, and the map $h: \hat{A}_{I} \rightarrow \widehat{A / I}$ obtained by passing to the quotient is a homeomorphism [15, Sect. 3.2]. Then $A / I$ is nontrivial and $\widehat{A / I}$ contains no isolated points. By Theorem 2.5 above and Corollary 3.19 of [26] the elements of of $\operatorname{Rep}(A / I, \mathcal{H})$ up to unitary equivalence do not admit classification by countable structures. Since $\operatorname{Rep}(A / I, \mathcal{H})$ can be view as the closed set of all representations in $\operatorname{Rep}(A, \mathcal{H})$ which factor through $A / I$, we obtain the theorem.

Finally we turn to the problem of classifying irreducible representations. By Glimm's theorem (see Section 6.8 of [41]), a separable $C^{*}$-algebra is of type I if and only if the Mackey Borel structure on $\hat{A}$ is standard. This is strengthened by the following result, which was shown by Hjorth for countable discrete groups [25] by a different argument that can also be applied to our more general setting.

Theorem 2.8. Let $A$ be a separable non-type $I C^{*}$-algebra. Then the irreducible representations of $A$ on $\mathcal{H}$ up to unitary equivalence do not admit classification by countable structures.

Proof. Since $A$ is not of type I, by Glimm's theorem [41, Thm. 6.8.7] there exists an essential irreducible representation $\pi$ of $A$ on $\mathcal{H}$. Set $B=A / \operatorname{ker}(\pi)$. Write $\operatorname{Irr}(B, \mathcal{H})$ for the set of irreducible representations in $\operatorname{Rep}(B, \mathcal{H})$, which is a dense $G_{\delta}$ by Lemma 2.1 and [15, Prop. 3.7.4]. Since $B$ admits a faithful irreducible representation its spectrum $\hat{B}$ contains no isolated points and so by Theorem 2.5 the action of $\mathcal{U}(\mathcal{H})$ on $\operatorname{Rep}(B, \mathcal{H})$, and hence also on $\operatorname{Irr}(B, \mathcal{H})$, is generically turbulent. We may view $\operatorname{Irr}(B, \mathcal{H})$ as the closed 
subset of $\operatorname{Irr}(A, \mathcal{H})$ consisting of those irreducible representations of $A$ which factor through $B$, and so we conclude by Corollary 3.19 of [26] that the irreducible representations of $A$ do not admit classification by countable structures.

\section{REPRESENTATIONS OF GROUPS}

Here we record some consequences of Section 2 for unitary group representations. Let $G$ be a second countable locally compact group. We write $\operatorname{Rep}(G, \mathcal{H})$ for $\operatorname{Rep}\left(C^{*}(G), \mathcal{H}\right)$, and we denote by $\operatorname{WM}(G, \mathcal{H})$ the subset of representations in $\operatorname{Rep}(G, \mathcal{H})$ which are weak mixing, i.e., which have no nonzero finite-dimensional subrepresentations. The subset $\mathrm{WM}(G, \mathcal{H})$ is a $G_{\delta}[4$ and it is dense precisely when $G$ has property $\mathrm{T}$ [35, 31].

As mentioned previously, Theorem 2.8 specializes in the group setting to the following theorem of Hjorth [25]. Actually Hjorth proved the result in the discrete case but his argument works more generally using Glimm's theorem.

Theorem 3.1 (Hjorth). Suppose that $G$ is not of type I. Then the irreducible representations of $G$ do not admit classification by countable structures.

By Theorem 2.7, the orbit equivalence relation of $\mathcal{U}(\mathcal{H})$ acting on $\operatorname{Rep}(G, \mathcal{H})$ either does not admit classification by countable structures or is smooth according to whether $\hat{G}$ is uncountable or countable. As an example of Fell illustrates, it is possible for a noncompact second countable locally compact group to have countable dual (see Section IV of [1]). On the other hand, we can deduce nonclassifiability for countable structures when $G$ is countably infinite or a noncompact separable Lie group, and in these cases we can furthermore restrict to representations that are weakly contained in $\lambda_{G}$, as we now explain.

We write $\hat{G}_{\lambda}$ for the reduced dual of $G$, i.e., the closed set of all elements in $\hat{G}$ which are weakly contained in $\lambda_{G}$. We denote by $\operatorname{Rep}_{\lambda}(G, \mathcal{H})$ the closed set of representations in $\operatorname{Rep}(G, \mathcal{H})$ which are weakly contained in $\lambda_{G}$. By Proposition H.2 in 31 this is equal to the closure of the orbit of $\lambda_{G}^{\oplus \mathbb{N}}$ viewed as a representation on $\mathcal{H}$ via some unitary equivalence. We write $\mathrm{WM}_{\lambda}(G, \mathcal{H})$ for $\operatorname{WM}(G, \mathcal{H}) \cap \operatorname{Rep}_{\lambda}(G, \mathcal{H})$.

The following is well known.

Lemma 3.2. Suppose that $G$ is countably infinite. Then the left regular representation $\lambda_{G}$ has no irreducible subrepresentations and $\hat{G}_{\lambda}$ has no isolated points.

Proof. By Corollary 5.12 of [44], $G$ has no square-integrable irreducible representations and thus, since square-integrability for a representation is equivalent to being a subrepresentation of $\lambda_{G}, G$ has no irreducible subrepresentations. Consequently $\hat{G}_{\lambda}$ has no isolated points by Corollary 1.9 of [49].

Theorem 3.3. Suppose that $G$ is countably infinite. Then the action of $\mathcal{U}(\mathcal{H})$ on $\operatorname{Rep}_{\lambda}(G, \mathcal{H})$ is generically turbulent. Furthermore, the action is turbulent precisely when $C_{\lambda}^{*}(G)$ is simple.

Proof. The result then follows by Lemma 3.2 and Theorem 2.5 .

Combining Theorem 3.3 with Corollary 3.19 of [26] yields:

Theorem 3.4. Suppose that $G$ is countably infinite. Then the elements of $\operatorname{Rep}_{\lambda}(G, \mathcal{H})$ up to unitary equivalence do not admit classification by countable structures. 
Remark 3.5. In Theorems 3.3 and 3.4 we can replace $\operatorname{Rep}_{\lambda}(G, \mathcal{H})$ by $\mathrm{WM}_{\lambda}(G, \mathcal{H})$, as follows from the following fact, which we record as a proposition for future reference.

Proposition 3.6. For a second countable locally compact group $G, \mathrm{WM}_{\lambda}(G, \mathcal{H})$ is a dense $G_{\delta}$ subset of $\operatorname{Rep}_{\lambda}(G, \mathcal{H})$.

Proof. If $G$ is amenable then $\operatorname{Rep}_{\lambda}(G, \mathcal{H})=\operatorname{Rep}(G, \mathcal{H})$ and, by Theorem 2.5 of [4], $\operatorname{WM}(G, \mathcal{H})$ is a dense $G_{\delta}$ subset of $\operatorname{Rep}(G, \mathcal{H})$. If $G$ is nonamenable then every element of $\operatorname{Rep}_{\lambda}(G, \mathcal{H})$ is weakly mixing, for if an element of $\operatorname{Rep}_{\lambda}(G, \mathcal{H})$ contains a finite-dimensional subrepresentation $\pi$ then $\pi \otimes \bar{\pi}$ contains the trivial representation and is weakly contained in $\lambda_{G}$, contradicting nonamenability.

Theorem 2.5 of [1] asserts that a separable Lie group whose reduced dual is countable must be compact, and so by Theorem 2.7 we can conclude the following.

Theorem 3.7. Let $G$ be a separable noncompact Lie group. Then the elements of $\operatorname{Rep}_{\lambda}(G, \mathcal{H})$ up to unitary equivalence do not admit classification by countable structures.

\section{TRACE-PRESERVING ACTIONS}

Let $M$ be a von Neumann algebra with separable predual, and let $\tau$ be a faithful normal tracial state on $M$. We write $\|\cdot\|_{2}$ for the $\tau$-norm on $M$, i.e., $\|a\|_{2}=\tau\left(a^{*} a\right)^{1 / 2}$. Let $G$ be a second countable locally compact group. We denote by $\operatorname{Act}(G, M, \tau)$ the Polish space of continuous $\tau$-preserving actions of $G$ on $M$ whose topology has as a basis the sets

$$
Y_{\alpha, K, \Omega, \varepsilon}=\left\{\beta \in \operatorname{Act}(G, M, \tau):\left\|\beta_{s}(a)-\alpha_{s}(a)\right\|_{2}<\varepsilon \text { for all } s \in K \text { and } a \in \Omega\right\}
$$

where $\alpha \in \operatorname{Act}(G, M, \tau), K$ is a compact subset of $G, \Omega$ is a finite subset of $M$, and $\varepsilon>0$. We equip the group $\operatorname{Aut}(M, \tau)$ of $\tau$-preserving automorphisms of $M$ with the Polish topology which has as a basis the sets

$$
Z_{\alpha, \Omega, \varepsilon}=\left\{\beta \in \operatorname{Aut}(R, G):\|\beta(a)-\alpha(a)\|_{2}<\varepsilon \text { for all } a \in \Omega\right\}
$$

where $\alpha \in \operatorname{Aut}(R, G), \Omega$ is a finite subset of $M$, and $\varepsilon>0$. We have a continuous action of $\operatorname{Aut}(M, \tau)$ on $\operatorname{Act}(G, M, \tau)$ given by $(\gamma \cdot \alpha)_{s}(a)=\left(\gamma \circ \alpha_{s} \circ \gamma^{-1}\right)(a)$ for all $s \in G, a \in A$, $\gamma \in \operatorname{Aut}(M, \tau)$, and $\alpha \in \operatorname{Act}(G, M, \tau)$.

For every $\alpha \in \operatorname{Act}(G, M, \tau)$ we write $\kappa_{\alpha}$ for the associated the unitary representation of $G$ on the GNS Hilbert space $L^{2}(M, \tau)$ given by $\pi_{\sigma}(s) a \xi=\alpha_{s}(a) \xi$ for $a \in M$, where $\xi$ is the canonical cyclic vector and $M$ is viewed as acting on $L^{2}(M, \tau)$ via left multiplication. The restriction of $\kappa_{\alpha}$ to $L^{2}(M, \tau) \ominus \mathbb{C} \mathbf{1}$ will be denoted $\kappa_{\alpha, 0}$.

An action $\alpha \in \operatorname{Act}(G, M, \tau)$ is said to be ergodic if $\kappa_{\alpha, 0}$ is ergodic (i.e., if $\kappa_{\alpha, 0}$ has no nonzero $G$-invariant vectors), and weakly mixing if $\kappa_{\alpha, 0}$ is weakly mixing (i.e., if $\kappa_{\alpha, 0}$ has no nonzero finite-dimensional subrepresentations). See [47, App. D] for some standard characterizations of weak mixing for actions. We write $\operatorname{WM}(G, M, \tau)$ for the set of weakly mixing actions in $\operatorname{Act}(G, M, \tau)$. This is a $G_{\delta}$ set, as the proof of Proposition 2.3 in [4] shows. 
4.1. Freeness. An automorphism $\theta$ of a von Neumann algebra $M$ is said to be properly outer if for every nonzero $\theta$-invariant projection $p$ the restriction of $\theta$ to $p M p$ is not inner [29] 46, Defn. XVII.1.1]. We may equivalently quantify over $\theta$-invariant projections in the centre of $M$ (see the comment after Theorem XVII.1.2 in [46]). An action $\alpha$ of $G$ on $M$ is said to be free if $\alpha_{s}$ is properly outer for every $s \in G \backslash\{e\}$. In the commutative case this is equivalent to the usual definition for actions on probability spaces. The aim of this subsection is to show that when $G$ is countable the free actions form a $G_{\delta}$ subset of $\operatorname{Act}(G, M, \tau)$, which was observed by Glasner and King for measure-preserving tranformations of a standard atomless probability space [20. To this end we will establish in Lemma 4.2 some characterizations of proper outerness in the tracial case. Compare [9, Thm. 1.2.1] and [42, Thm. 3.3].

An example of a noncommutative free action in the case that $G$ is countably infinite is the Bernoulli shift $\beta$ on the weak operator closure of $M_{n}^{\otimes G}$ (with $n \geq 2$ ) in the tracial representation, which is isomorphic to $R$. The freeness of $\beta$ can be seen as follows. If $s$ is an element of $G$ of infinite order then it is well known and easy to check that the automorphism $\beta_{s}$ is mixing and in particular ergodic, in which case $\beta_{s}$ is not inner, for otherwise any unitary witnessing the innerness would be different from 1 and fixed by $\beta_{s}$. If $s$ is an element of $G \backslash\{e\}$ of finite order then $\beta_{s}$ is conjugate to an automorphism as in the statement of the following proposition and hence is not inner. Thus $\beta$ is free.

Proposition 4.1. Let $n \geq 2$ be an integer and let $\alpha$ be an automorphism of $M_{n}$ which is not the identity. Let $\theta$ be the extension of $\alpha^{\otimes \mathbb{N}}$ on the weak operator closure $R$ of $M_{n}^{\otimes \mathbb{N}}$ in the tracial representation. Then $\theta$ is not inner.

Proof. We can express $\alpha$ as $\operatorname{Ad} u$ for some unitary $u \in M_{n}$. Suppose that $\theta$ is inner. Then $\beta=\operatorname{Ad} v$ for some unitary $v \in R$. Let $k \geq 1$. Then $\theta$ restricts to $\operatorname{Ad} u^{\otimes[1, k]}$ on $M_{n}^{\otimes[1, k]}$. Denote by $E_{k}$ the trace-preserving conditional expectation of $R$ onto $M_{n}^{\otimes[1, k]}$. For every $a \in M_{n}^{\otimes[1, k]}$, from $\left(u^{\otimes[1, k]}\right) a\left(u^{\otimes[1, k]}\right)^{*} v=\theta(a) v=v a$ we obtain $\left(u^{\otimes[1, k]}\right) a\left(u^{\otimes[1, k]}\right)^{*} E_{k}(v)=$ $E_{k}(v) a$. Then $\left(u^{\otimes[1, k]}\right)^{*} E_{k}(v)$ is in the centre of $M_{n}^{\otimes[1, k]}$ and hence $E_{k}(v)=\lambda_{k}\left(u^{\otimes[1, k]}\right)$ for some $\lambda_{k} \in \mathbb{C}$. As $k \rightarrow \infty,\left|\lambda_{k}\right|=\left\|\lambda_{k}\left(u^{\otimes[1, k]}\right)\right\|_{2}=\left\|E_{k}(v)\right\|_{2} \rightarrow\|v\|_{2}=1$. We also have $E_{k}(v)=E_{k}\left(E_{k+1}(v)\right)=E_{k}\left(\lambda_{k+1}\left(u^{\otimes[1, k+1]}\right)\right)=\operatorname{tr}(u) \lambda_{k+1}\left(u^{\otimes[1, k]}\right)$, where tr denotes the normalized trace on $M_{n}$. Thus $\lambda_{k}=\operatorname{tr}(u) \lambda_{k+1}$. It follows that $|\operatorname{tr}(u)|=1$. By the Cauchy-Schwarz inequality we get $u \in \mathbb{C}$. This implies that $\alpha=\operatorname{Ad} u$ is trivial, contradicting our assumption. Therefore $\beta$ is not inner.

The equivalence of the first two conditions in the following lemma is due to Connes, who proved it for arbitrary automorphisms of a countably decomposable von Neumann algebra [9, Thm. 1.2.1].

Lemma 4.2. Let $M$ be a von Neumann algebra with faithful normal tracial state $\tau$. Let $\theta \in \operatorname{Aut}(M, \tau)$. Let $0<\lambda \leq 1 / 3$ and $0<\varepsilon<1$. Let $S$ be a $\tau$-norm dense subset of the set of all nonzero projections in $M$. Then the following are equivalent:

(1) $\theta$ is properly outer,

(2) for every nonzero projection $p \in M$ there is a nonzero projection $q \in M$ with $q \leq p$ and $\|q \theta(q)\|<\varepsilon$, 
(3) for every nonzero projection $p \in M$ there is a nonzero projection $q \in M$ with $q \leq p$ and $\|q \theta(q)\|_{2}<\varepsilon\|q\|_{2}$,

(4) for every $p \in S$ there is a projection $q \in M$ with $q \leq p$, $\|q \theta(q)\|_{2}<\varepsilon\|q\|_{2}$, and $\tau(q) \geq \lambda \tau(p)$

(5) for every nonzero projection $p \in M$ there is an $x \in p M p$ with $\|x-\theta(x)\|_{2}>$ $(1-\varepsilon)\|x\|_{2}$

(6) for every nonzero $\theta$-invariant projection $p \in M$ there is an $x \in p M p$ with $\| x-$ $\theta(x)\left\|_{2}>(1-\varepsilon)\right\| x \|_{2}$.

Proof. $(1) \Rightarrow(2)$. This is part of [9, Thm. 1.2.1].

$(2) \Rightarrow(3)$. With $q$ as in (2) we have

$$
\begin{aligned}
\|q \theta(q)\|_{2}^{2} & =\|\theta(q) q\|_{2}^{2}=\tau(q q \theta(q) q q) \leq \tau(q\|q \theta(q) q\| q) \\
& =\|q \theta(q) q\| \tau(q)=\|q \theta(q)\|^{2}\|q\|_{2}^{2}<\varepsilon^{2}\|q\|_{2}^{2} .
\end{aligned}
$$

$(3) \Rightarrow(5)$. This follows by observing that $q$ as in (3) satisfies

$$
\|q-\theta(q)\|_{2} \geq\|q-q \theta(q)\|_{2} \geq\|q\|_{2}-\|q \theta(q)\|_{2}>(1-\varepsilon)\|q\|_{2} .
$$

$(5) \Rightarrow(6)$. Trivial.

$(6) \Rightarrow(1)$. Suppose that $\theta$ is not properly outer. Then there exists a nonzero $\theta$-invariant projection $e \in M$ such that $\left.\theta\right|_{e M e}$ is equal to $\operatorname{Ad} u$ for some unitary $u \in e M e$. Let $p$ be a nonzero spectral projection of $u$ such that $\|u p-t p\|<(1-\varepsilon) / 2$ for some $t \in \mathbb{C}$ with $|t|=1$. Then $p$ is $\theta$-invariant, and for every $x \in p M p$ we have

$$
\begin{aligned}
\|x-\theta(x)\|_{2} & =\left\|(t p) x(\bar{t} p)-u p x p u^{*}\right\|_{2} \\
& \leq\|(t p-u p) x(\bar{t} p)\|_{2}+\left\|u p x\left(\bar{t} p-p u^{*}\right)\right\|_{2} \\
& \leq\|t p-u p\|\|x\|_{2}\|\bar{t} p\|+\|u p\|\|x\|_{2}\left\|\bar{t} p-p u^{*}\right\| \\
& \leq(1-\varepsilon)\|x\|_{2},
\end{aligned}
$$

contradicting (6).

$(3) \Rightarrow(4)$. Let $p \in S$. Let $\left\{q_{j}\right\}_{j \in J}$ be a maximal family of subprojections of $p$ such that $q_{j} \perp q_{k}$ and $q_{j} \perp \theta\left(q_{k}\right)$ for all distinct $j, k \in J$ and $\left\|q_{j} \theta\left(q_{j}\right)\right\|_{2}<\varepsilon\left\|q_{j}\right\|_{2}$ for each $j \in J$. Set $q=\sum_{j \in J} q_{j}$. Then

$$
\begin{aligned}
\|q \theta(q)\|_{2}^{2} & =\tau(q \theta(q) q)=\sum_{j} \tau\left(q_{j} \theta\left(q_{j}\right) q_{j}\right)=\sum_{j \in J}\left\|q_{j} \theta\left(q_{j}\right)\right\|_{2}^{2} \\
& <\varepsilon^{2} \sum_{j \in J}\left\|q_{j}\right\|_{2}^{2}=\varepsilon^{2}\|q\|_{2}^{2},
\end{aligned}
$$

and hence $\|q \theta(q)\|_{2}<\varepsilon\|q\|_{2}$. We claim that $p \precsim \theta^{-1}(q) \vee q \vee \theta(q)$. Suppose that this is not true. Since the range projection $p^{\prime}$ of $p\left(\theta^{-1}(q) \vee q \vee \theta(q)\right)$ is equivalent to the orthogonal complement of its kernel projection, which is a subprojection of $\theta^{-1}(q) \vee q \vee \theta(q)$, we see that $p^{\prime} \neq p$. By (3) we can find a projection $e \in M$ with $e \leq p-p^{\prime}$ and $\|e \theta(e)\|_{2}<\varepsilon\|e\|_{2}$. Then $e\left(\theta^{-1}(q) \vee q \vee \theta(q)\right)=e p\left(\theta^{-1}(q) \vee q \vee \theta(q)\right)=0$ and hence we can add $e$ to the family $\left\{p_{j}\right\}_{j \in J}$ to get a larger family, which is a contradiction. Thus $p \precsim \theta^{-1}(q) \vee q \vee \theta(q)$ as claimed. Note that for any projections $e_{1}, e_{2} \in M$ we have $e_{1} \vee e_{2}=e_{1}+e_{3}$ for some 
$e_{3} \sim e_{2}-e_{1} \wedge e_{2}$ and hence $\tau\left(e_{1} \vee e_{2}\right) \leq \tau\left(e_{1}\right)+\tau\left(e_{2}\right)$. Thus

$$
\tau(p) \leq \tau\left(\theta^{-1}(q) \vee q \vee \theta(q)\right) \leq \tau\left(\theta^{-1}(q)\right)+\tau(q)+\tau(\theta(q))=3 \tau(q) .
$$

$(4) \Rightarrow(5)$. With $\varepsilon$ as in (4), let us show that (5) holds for any given $\varepsilon^{\prime}$ satisfying $1>$ $\varepsilon^{\prime}>\varepsilon$. Take a $p^{\prime} \in S$ with $\left\|p-p^{\prime}\right\|_{2}<\delta$ for $\delta$ to be determined later. By (4) we can find a projection $q^{\prime} \in M$ with $q^{\prime} \leq p^{\prime}$ and $\left\|q^{\prime} \theta\left(q^{\prime}\right)\right\|_{2}<\varepsilon\left\|q^{\prime}\right\|_{2}$ and $\tau\left(q^{\prime}\right) \geq \lambda \tau\left(p^{\prime}\right)$. As in the proof of $(3) \Rightarrow(5)$ we have $\left\|q^{\prime}-\theta\left(q^{\prime}\right)\right\|_{2}>(1-\varepsilon)\left\|q^{\prime}\right\|_{2}$. Set $x=p q^{\prime} p \in p M p$. Then

$$
\left\|x-q^{\prime}\right\|_{2}=\left\|p q^{\prime} p-p^{\prime} q^{\prime} p^{\prime}\right\|_{2} \leq 2\left\|p-p^{\prime}\right\|_{2}<2 \delta
$$

and hence

$$
\|x\|_{2}>\left\|q^{\prime}\right\|_{2}-2 \delta \geq \lambda^{1 / 2}\left\|p^{\prime}\right\|_{2}-2 \delta>\lambda^{1 / 2}(\|p\|-\delta)-2 \delta \geq \frac{4}{\varepsilon^{\prime}-\varepsilon} \delta,
$$

granted $\delta$ is chosen small enough. Therefore

$$
\begin{aligned}
\|x-\theta(x)\|_{2} & \geq\left\|q^{\prime}-\theta\left(q^{\prime}\right)\right\|_{2}-2\left\|x-q^{\prime}\right\|_{2}>(1-\varepsilon)\left\|q^{\prime}\right\|_{2}-4 \delta \\
& \geq(1-\varepsilon)\|x\|_{2}-4 \delta \geq\left(1-\varepsilon^{\prime}\right)\|x\|_{2} .
\end{aligned}
$$

Lemma 4.3. Let $M$ be a von Neumann algebra with separable predual and faithful normal tracial state $\tau$. Then for a countable $G$ the set of free actions in $\operatorname{Act}(G, M, \tau)$ is a $G_{\delta}$.

Proof. Take an increasing sequence $K_{1} \subseteq K_{2} \subseteq \ldots$ of finite subsets of $G$ whose union is $G \backslash\{e\}$, and an increasing sequence $\Omega_{1} \subseteq \Omega_{2} \subseteq \ldots$ of finite sets of nonzero projections in $M$ whose union is $\tau$-norm dense in the set of all nonzero projections in $M$. For every $n \in \mathbb{N}$ write $W_{n}$ for the open set of all $\alpha \in \operatorname{Act}(G, M, \tau)$ such that for every $s \in K_{n}$ and $p \in \Omega_{n}$ there is a projection $q \leq p$ satisfying $\left\|q \alpha_{s}(q)\right\|_{2}<\|q\|_{2} / 3$ and $\|q\|_{2} \geq\|p\|_{2} / 2$. Then $W:=\bigcap_{n=1}^{\infty} W_{n}$ is a $G_{\delta}$, and it consists precisely of the free actions by Lemma 4.2 ,

4.2. Nonclassifiability by countable structures. The proof of the theorems in this subsection will require Gaussian and Bogoliubov actions (cf. the proof of Theorem 13.7 in [31]), and so we will begin by briefly recalling these constructions.

Gaussian Hilbert spaces provide a standard means for producing actions from representations (see Appendix E of [31] or Appendix A.7 of [3]). Let $\mathcal{H}$ be a separable infinite-dimensional real Hilbert space. Write $\nu$ for the standard Gaussian measure $(2 \pi)^{-1 / 2} e^{-x^{2} / 2} d x$ on $\mathbb{R}$. Fix an isometric isomorphism $\varphi: \mathcal{H} \rightarrow \mathcal{H}^{: 1:}$ where $\mathcal{H}^{: 1:}$ is the closed subspace of $L_{2}\left(\mathbb{R}^{\mathbb{N}}, \nu^{\mathbb{N}}\right)$ spanned by the coordinate projections, i.e., the first Wiener chaos. Then associated to each orthogonal operator $S$ on $\mathcal{H}$ is a unique automorphism in $\operatorname{Aut}\left(\mathbb{R}^{\mathbb{N}}, \nu^{\mathbb{N}}\right)$ whose Koopman operator restricts on $\mathcal{H}: 1:$ to $\varphi \circ S \circ \varphi^{-1}$. Thus from every representation in $\operatorname{Rep}(G, \mathcal{H})$ we obtain a $\nu^{\mathbb{N}}$-preserving action of $G$ on $\left(\mathbb{R}^{\mathbb{N}}, \nu^{\mathbb{N}}\right)$, called a Gaussian action, and up to conjugacy this action depends only on the orthogonal equivalence class of the representation. The orthogonal Koopman representation for the Gaussian action associated to a $\pi \in \operatorname{Rep}(G, \mathcal{H})$ is orthogonally equivalent to the direct sum $\bigoplus_{n=0}^{\infty} S^{n} \pi$ of all symmetric tensor powers of $\pi$.

By the Gaussian action associated to a unitary representation of $G$ on a separable infinite-dimensional complex Hilbert space $\mathcal{H}$ we mean the Gaussian action obtained from the induced orthogonal representation of $G$ on the realification of $\mathcal{H}$. From the description 
of the orthogonal Koopman representation in the latter case we see that if $\pi$ is weakly mixing then so is $\beta$, and if $\pi$ is weakly contained in $\lambda_{G}$ then so is $\kappa_{\beta, 0}$. These two facts will be used in the proof of Theorem 4.4.

Bogoliubov actions are constructed as follows (see [6] for a general reference). Let $\mathcal{H}$ be a separable infinite-dimensional Hilbert space. The CAR algebra $A(\mathcal{H})$ is defined as the unique, up to ${ }^{*}$-isomorphism, unital $C^{*}$-algebra generated by operators $a(\xi)$ for $\xi \in \mathcal{H}$ such that the map $\xi \mapsto a(\xi)^{*}$ is linear and the anticommutation relations

$$
\begin{aligned}
a(\xi) a(\zeta)^{*}+a(\zeta)^{*} a(\xi) & =\langle\zeta, \xi\rangle \mathbf{1}_{A(\mathcal{H})}, \\
a(\xi) a(\zeta)+a(\zeta) a(\xi) & =0,
\end{aligned}
$$

hold for all $\xi, \zeta \in \mathcal{H}$. The $C^{*}$-algebra $A(\mathcal{H})$ is ${ }^{*}$-isomorphic to the type $2^{\infty}$ UHF algebra and has a unique tracial state [6]. The weak operator closure (equivalently, strong operator closure) of $A(\mathcal{H})$ in the tracial representation is isomorphic to the hyperfinite $\mathrm{II}_{1}$ factor [5, Prop. III.3.4.6] and we will write it as $W(\mathcal{H})$. Corresponding to a unitary operator $U$ on $\mathcal{H}$ is the Bogoliubov automorphism of $A(\mathcal{H})$ determined by $a(\xi) \mapsto a(U \xi)$ for $\xi \in \mathcal{H}$. A representation $\pi \in \operatorname{Rep}(G, \mathcal{H})$ gives rise via Bogoliubov automorphisms to a continuous action of $G$ on $A(\mathcal{H})$. Every continuous action of $G$ on the CAR algebra by *-automorphisms is trace-preserving and hence extends to a continuous action on $W(\mathcal{H})$. We refer to these actions as Bogoliubov actions. The tracial state $\tau$ on $A(\mathcal{H})$ is determined by

$$
\tau\left(a^{*}\left(\xi_{1}\right) \cdots a^{*}\left(\xi_{n}\right) a\left(\zeta_{m}\right) \cdots a\left(\zeta_{1}\right)\right)=\delta_{n m} 2^{-n} \operatorname{det}\left(\left\langle\xi_{i}, \zeta_{j}\right\rangle\right)_{i, j} .
$$

The tracial GNS representation $\left(\pi_{\tau}, \mathcal{H}_{\tau}, \Omega_{\tau}\right)$ and the corresponding unitary implementation on $\mathcal{H}_{\tau}$ of a unitary representation $\sigma$ of $G$ on $\mathcal{H}$ can be described as follows (see Section 2 of [16] for more details and references). The antisymmetric Fock space $F(\mathcal{H})$ is defined as $\bigoplus_{n=0}^{\infty} \Lambda^{n} \mathcal{H}$ where $\Lambda^{n} \mathcal{H}$ is the $n$th antisymmetric tensor power of $\mathcal{H}$, which for $n=0$ is defined as $\mathbb{C}$ with unit vector $\Omega$. We have an irreducible representation $\rho$ of the CAR algebra on $F(\mathcal{H})$ determined by

$$
\rho\left(a(\xi)^{*}\right) \zeta_{1} \wedge \cdots \wedge \zeta_{n}=\xi \wedge \zeta_{1} \wedge \cdots \wedge \zeta_{n} .
$$

For a unitary operator $U$ on $\mathcal{H}$ we write $F(U)$ for the unitary operator on $F(\mathcal{H})$ which acts on $\Lambda^{n} \mathcal{H}$ as $U^{\otimes n}$ and as the identity on $\Omega$. Writing $\overline{\mathcal{H}}$ for the conjugate Hilbert space of $\mathcal{H}$ and $\xi \mapsto \bar{\xi}$ for the canonical antilinear isometry from $\mathcal{H}$ to $\overline{\mathcal{H}}$, we define a representation $\pi$ of $A(\mathcal{H})$ on $F(\mathcal{H}) \otimes_{2} F(\overline{\mathcal{H}})$ by

$$
\pi(a(\xi))=\frac{1}{\sqrt{2}}\left[\rho(a(\xi)) \otimes F(-\mathbf{1})+\mathbf{1} \otimes \rho\left(a(\bar{\xi})^{*}\right)\right] .
$$

It can then be checked that the vector state on $A(\mathcal{H})$ associated to $\Omega \otimes \Omega$ coincides with $\tau$, so that $\pi_{\tau}$ can be identified with a subrepresentation of $\pi$. Writing $\beta$ for the Bogoliubov action associated to $\sigma$ and $F(\sigma)$ for the unitary representation $s \mapsto F\left(\sigma_{s}\right)$ of $G$ on $F(\mathcal{H})$, we observe accordingly that the representation $\kappa_{\beta, 0}$ of $G$ on $\mathcal{H}_{\tau} \ominus \mathbb{C} \Omega_{\tau}$ arising from $\beta$ can be viewed as a subrepresentation of

$$
\left(F(\sigma)_{0} \otimes \mathbf{1}_{G}\right) \oplus\left(F(\sigma)_{0} \otimes F(\bar{\sigma})_{0}\right) \oplus\left(\mathbf{1}_{G} \otimes F(\bar{\sigma})_{0}\right)
$$

where $F(\pi)_{0}$ for a representation $\pi$ means the restriction of $F(\pi)$ to the orthogonal complement of $\mathbb{C} \Omega$. Since a tensor product of two representations is weakly contained in a 
tensor product of two other representations under the assumption of factorwise weak containment, we see that if $\sigma$ is weakly contained in $\lambda_{G}$ then so are $F(\sigma)_{0}$ and $F(\bar{\sigma})_{0}$ and hence so is $\kappa_{\beta, 0}$. Since weak mixing for representations is preserved under taking tensor products, we also observe that if $\sigma$ is weakly mixing then $\beta$ is weakly mixing (as can alternatively be seen using the formula for $\tau$ from above). Note also from the formula for $\tau$ that $\pi$ embeds as a subrepresentation of $\kappa_{\beta}$ via the map $\xi \mapsto \pi_{\tau}\left(\sqrt{2} a(\xi)^{*}\right) \Omega_{\tau}$ from $\mathcal{H}$ to $\mathcal{H}_{\tau}$. We will invoke these facts in the proof of Theorem 4.4 .

In the following results $M$ is assumed to be either $L_{\infty}(X, \tau)$ for some standard atomless probability space $(X, \tau)$ or the hyperfinite $\mathrm{II}_{1}$ factor $R$ with its unique faithful normal tracial state $\tau$. We write $\operatorname{Act}_{\lambda}(G, M, \tau)$ for the closed set of all $\alpha \in \operatorname{Act}(G, M, \tau)$ such that the associated representation $\kappa_{\alpha, 0}$ is weakly contained in the left regular representation. We also define

$$
\mathrm{WM}_{\lambda}(G, M, \tau)=\operatorname{WM}(G, M, \tau) \cap \operatorname{Act}_{\lambda}(G, M, \tau),
$$

which is a $G_{\delta}$ in $\operatorname{Act}(G, M, \tau)$, and write $\operatorname{FWM}_{\lambda}(G, M, \tau)$ for the set of free weakly mixing actions in $\operatorname{Act}_{\lambda}(G, M, \tau)$, which for countable $G$ is a $G_{\delta}$ by Lemma 4.3 .

To establish the following theorem we argue by contradiction following the scheme of the proof of Theorem 13.7 in 31].

Theorem 4.4. Suppose that $G$ is countably infinite. Then up to conjugacy the elements of $\mathrm{FWM}_{\lambda}(G, M, \tau)$ do not admit classification by countable structures.

Proof. Suppose that there does exist a Borel function $F$ from $\operatorname{FWM}_{\lambda}(G, M, \tau)$ to the space $\Theta_{L}$ of countable structures on some countable language $L$ such that $\alpha$ is conjugate to $\beta$ if and only if $F(\alpha) \cong F(\beta)$. By Theorem 3.3 and Proposition 3.6 there is a dense $G_{\delta}$ set $Z$ of weakly mixing representations in $\operatorname{Rep}_{\lambda}(G, \mathcal{H})$ on which the action of $\mathcal{U}(\mathcal{H})$ is turbulent. Fix an action $\sigma$ in $\operatorname{Act}_{\lambda}(G, M, \tau)$ which is free and weakly mixing, such as a Bernoulli shift (see Subsection 4.1). Then for every $\gamma \in \mathrm{WM}(G, M, \tau)$ the action $\sigma \otimes \gamma$ is weakly mixing and, by Corollary 1.12 of [29], free.

Recall from the discussion above that if $\pi$ is a weakly mixing representation in $\operatorname{Rep}_{\lambda}(G, \mathcal{H})$ then the associated Gaussian action (if $M=L_{\infty}(X, \tau)$ ) or the associated Bogoliubov action (if $M=R$ ) is contained in $\mathrm{WM}_{\lambda}(G, M, \tau)$. By Theorem 3.18 of [26] there exists a $K \in \Theta_{L}$ such that $F\left(\sigma \otimes \beta_{\pi}\right) \cong K$ for all $\pi$ in a comeager subset $Z_{0}$ of $Z$, where $\beta_{\pi}$ is the associated Gaussian or Bogoliubov action and $\sigma \otimes \beta_{\pi}$ is viewed as an action on $M$ via some fixed isomorphism. Thus there is an $\alpha \in \mathrm{WM}_{\lambda}(G, M, \tau)$ such that $\alpha$ is conjugate to $\sigma \otimes \beta_{\pi}$ for all $\pi \in Z_{0}$. But then $\kappa_{\alpha}$ contains every representation in $Z_{0}$, which is a contradiction because the set of representations in $\operatorname{Rep}_{\lambda}(G, \mathcal{H})$ which are disjoint from $\kappa_{\alpha}$ is a dense $G_{\delta}$ by Lemmas 2.4 and 3.2 and hence has nonempty intersection with $Z_{0}$.

The techniques of Hjorth using irreducible representations [25] 31, Thm. 13.7] can be applied to give nonclassifiability-by-countable-structures results for actions of any second countable locally compact group which is not compact and not type I. This excludes however many groups of interest, such as $\mathbb{R}^{d}$ and $\mathrm{SL}(2, \mathbb{R})$. The following theorem applies in particular to all noncompact separable Lie groups which are not amenable [1, Thm. 2.5] as well as many, if not all, which are amenable. To put the hypotheses into perspective, we remark as in Section 3 that Fell constructed an example of a noncompact second countable locally compact group with countable dual (see Section IV of [1]). 
For a closed set $C \subseteq \hat{G}$, we write $\operatorname{Rep}_{C}(G, \mathcal{H})$ for the closed set of representations in $\operatorname{Rep}(G, \mathcal{H})$ which are weakly contained in $C$, and $\mathrm{WM}_{C}(G, \mathcal{H})$ for $\operatorname{WM}(G, \mathcal{H}) \cap \operatorname{Rep}_{C}(G, \mathcal{H})$.

Theorem 4.5. Let $G$ be a second countable locally compact group such that either (i) $G$ is not amenable and $\hat{G}_{\lambda}$ is uncountable, or (ii) $G$ is amenable and the set of isolated points in $\hat{G}$ is not dense. Then up to conjugacy the elements of $\mathrm{WM}_{\lambda}(G, M, \tau)$ do not admit classification by countable structures.

Proof. Let $\mathcal{H}$ be a separable infinite-dimensional Hilbert space. Suppose first that $G$ is not amenable and $\hat{G}_{\lambda}$ is uncountable. Let $C$ be the closed set of condensation points of $\hat{G}_{\lambda}$, which is nonempty by the uncountability of the latter. Since $G$ is not amenable $\hat{G}_{\lambda}$ contains no finite-dimensional representations, and so $\mathrm{WM}_{C}(G, \mathcal{H})=\operatorname{Rep}_{C}(G, \mathcal{H})$. By Theorem 2.5 there is a dense $G_{\delta}$ set $Z \subseteq \mathrm{WM}_{C}(G, \mathcal{H})$ on which the action of $\mathcal{U}(\mathcal{H})$ is turbulent. Suppose that there exists a Borel function $F$ from $\mathrm{WM}_{\lambda}(G, M, \tau)$ to the space $\Theta_{L}$ of countable structures on some countable language $L$ such that $\alpha$ is conjugate to $\beta$ if and only if $F(\alpha) \cong F(\beta)$. Theorem 3.18 of [26] yields the existence of a $K \in \Theta_{L}$ such that $F\left(\beta_{\pi}\right) \cong K$ for all $\pi$ in a comeager subset $Z_{0}$ of $Z$, where $\beta_{\pi}$ is the associated Gaussian action if $M=L_{\infty}(X, \tau)$ or the associated Bogoliubov action on $W(\mathcal{H})$ if $M=R$ (which, as in the proof of Theorem 4.4, are both weakly mixing since $\pi$ is weakly mixing) and $\beta_{\pi}$ is viewed as an action on $M$ via some fixed isomorphism. Consequently there is an $\alpha \in \mathrm{WM}_{\lambda}(G, M, \tau)$ such that $\alpha$ is conjugate to $\beta_{\pi}$ for all $\pi \in Z_{0}$. Then $\kappa_{\alpha}$ contains every representation in $Z_{0}$, which is a contradiction because the set of representations in $\mathrm{WM}_{C}(G, \mathcal{H})$ which are disjoint from $\kappa_{\alpha}$ is a dense $G_{\delta}$ by Lemma 2.4 and hence has nonempty intersection with $Z_{0}$. Thus $\mathrm{WM}_{C}(G, M, \tau)$, and hence also $\mathrm{WM}_{\lambda}(G, M, \tau)$, does not admit classification by countable structures.

Suppose now that $G$ is amenable and the set of isolated points in $\hat{G}$ is not dense. Then $\operatorname{WM}(G, \mathcal{H})=\mathrm{WM}_{\lambda}(G, \mathcal{H})$ and this set is a dense $G_{\delta}$ in $\operatorname{Rep}(G, \mathcal{H})$ [4, Thm. 2.5]. Then by Theorem 2.5 there is a dense $G_{\delta}$ set $Z \subseteq \mathrm{WM}(G, \mathcal{H})$ on which the action of $\mathcal{U}(\mathcal{H})$ is turbulent. Using this $Z$ we can now carry out an argument by contradiction as in the first paragraph to obtain the desired conclusion.

Remark 4.6. Conjugacy can be replaced by unitary equivalence in the statement of each of the theorems in this subsection, as is clear from the proofs.

\section{Turbulence And aCtions on the hyperfinite $\mathrm{II}_{1}$ FACtor}

Our main goal in this section is to establish turbulence in the space of free actions of a countably infinite amenable group on the hyperfinite $\mathrm{II}_{1}$ factor $R$. In Subsection 5.1 we show the meagerness of orbits in the space of actions of a countably infinite amenable group on $R$ by developing a noncommutative version of an entropy and disjointness argument of Foreman and Weiss [17. In Subsection 5.2 we show for general second countable locally compact $G$ how to construct a $G$-action on $R$ which has dense orbit and is a turbulent point, and then deduce turbulence in the space of free actions when $G$ is countably infinite and amenable by applying a theorem of Ocneanu.

As usual, $G$ is assumed to be a second countable locally compact group, subject to extra hypotheses as required. In this section $\tau$ will invariably denote the unique normal tracial state on $R$. We write $\operatorname{Aut}(R)$ for the automorphism group of $R$ and $\operatorname{Act}(G, R)$ 
for the set of continuous actions of $G$ on $R$. We regard these as Polish spaces under the topology defined in Section 4 , where we have dropped $\tau$ in the notation since every action on $R$ is $\tau$-preserving. The set of free actions in $\operatorname{Act}(G, R)$ will be written $\operatorname{Fr}(G, R)$. We will require the following two lemmas relating to freeness.

Lemma 5.1. Suppose that $G$ is countable. Then $\operatorname{Fr}(G, R)$ is a dense $G_{\delta}$ subset of $\operatorname{Act}(G, R)$.

Proof. By Lemma 4.3 we need only show the density. Find a free action $\beta$ in $\operatorname{Act}(G, R)$. If $G$ is infinite we may take a Bernoulli shift (see the first part of Subsection 4.1), while if $G$ is finite then we may take an embedding $\varphi$ of $G$ into the unitary group of $M_{n}$ for some $n$ and then use $s \mapsto(\operatorname{Ad} \varphi(s))^{\otimes \mathbb{N}}$ by Proposition 4.1. Take a dense sequence $\left\{\alpha_{i}\right\}_{i=1}^{\infty}$ in $\operatorname{Act}(G, R)$ and let $\alpha$ be an element of $\operatorname{Act}(G, R)$ which is conjugate to $\beta \otimes \bigotimes_{i=1}^{\infty} \alpha_{i} \in \operatorname{Act}\left(G, R \bar{\otimes} R^{\bar{\otimes} \mathbb{N}}\right)$. Then the orbit of $\alpha$ is dense in $\operatorname{Act}(G, R)$, as can be established using an argument as in the proof of Lemma 3.5 in 35 . Moreover, $\alpha$ is free since the tensor product of a free action with any other action is free [29, Cor. 1.12], completing the proof.

Lemma 5.2. Suppose that $G$ is countable and amenable. Then every free action in $\operatorname{Act}(G, R)$ has dense orbit.

Proof. A theorem of Ocneanu shows that if $G$ is a countable amenable group then given free actions $\alpha, \beta \in \operatorname{Act}(G, R)$, a finite set $K \subseteq G$, and an $\varepsilon>0$ there exist a $\theta \in \operatorname{Aut}(R)$ and unitaries $u_{s} \in R$ for $s \in K$ such that, for every $s \in K,\left\|u_{s}-1\right\|_{2}<\varepsilon$ and $\alpha_{s}=$ $\theta \circ\left(\operatorname{Ad} u_{s}\right) \circ \beta \circ \theta^{-1}$ (see Section 1.4 of [38]). Consequently the orbit of every free action is dense in $\operatorname{Fr}(G, R)$, and so by Lemma [5.1] we obtain the result.

5.1. Entropy, disjointness, and meagerness of orbits. We will use the entropy of Connes-Narnhofer-Thirring 11 as applied to actions of discrete amenable groups on $R$. For a general reference on CNT entropy see [37]. A channel is a u.c.p. (unital completely positive) map $\gamma: B \rightarrow R$ where $B$ is a finite-dimensional $C^{*}$-algebra. Given channels $\gamma_{i}: B \rightarrow R$ for $i=1, \ldots, n$ we write $H_{\tau}\left(\gamma_{1}, \ldots, \gamma_{n}\right)$ for the supremum of the entropies of the Abelian models for $\gamma_{1}, \ldots, \gamma_{n}$ (see Section III of [11]). By Theorem IV.3 of [11] the function $H_{\tau}(\cdot)$ is continuous with respect to the trace norm in the sense that, given a finite-dimensional $C^{*}$-algebra $B$, for every $\varepsilon>0$ there is a $\delta>0$ such that, for all $n \in \mathbb{N}$, if $\gamma_{i}, \gamma_{i}^{\prime}: B \rightarrow R$ are channels with $\sup _{\|a\| \leq 1}\left\|\gamma_{i}^{\prime}(a)-\gamma_{i}(a)\right\|_{2}<\delta$ for $i=1, \ldots, n$ then

$$
\left|H_{\tau}\left(\gamma_{1}^{\prime}, \ldots, \gamma_{n}^{\prime}\right)-H_{\tau}\left(\gamma_{1}, \ldots, \gamma_{n}\right)\right|<n \varepsilon .
$$

Suppose now that $G$ is discrete and amenable and let $\alpha \in \operatorname{Act}(G, R)$. We define $h_{\tau}(\gamma, \alpha)$ as the limit of

$$
\frac{1}{|F|} H_{\tau}\left(\left(\alpha_{s} \circ \gamma\right)_{s \in F}\right)
$$

as $F$ becomes more and more invariant. This limit exists by subadditivity [36, Theorem 6.1] [33, Proposition 3.22]. The CNT entropy $h_{\tau}(\alpha)$ of $\alpha$ is then defined as the supremum of $h_{\tau}(\gamma, \alpha)$ over all channels $\gamma: B \rightarrow R$.

Lemma 5.3. Suppose that $G$ is countably infinite and amenable. Then the set of $\alpha \in$ $\operatorname{Act}(G, R)$ with $h_{\tau}(\alpha)=0$ is a dense $G_{\delta}$. 
Proof. It suffices to show that, given an $\varepsilon>0$, the set $S_{\varepsilon}$ of actions in $\operatorname{Act}(G, R)$ with entropy at most $\varepsilon$ is a dense $G_{\delta}$, since the collection of zero entropy actions is equal to $\bigcap_{n=1}^{\infty} S_{1 / n}$. Take a Følner sequence $\left\{F_{k}\right\}_{k=1}^{\infty}$ in $G$ and finite-dimensional subfactors $N_{1} \subseteq N_{2} \subseteq \ldots$ of $R$ with trace norm dense union. Let $\gamma_{n}: N_{n} \hookrightarrow R$ be the inclusion map for each $n \in \mathbb{N}$. Then for all $n, m, l \in \mathbb{N}$ the set $S_{\varepsilon}(n, m, l)$ of all $\alpha \in \operatorname{Act}(G, R)$ such that

$$
H_{\tau}\left(\left(\alpha_{s} \circ \gamma_{n}\right)_{s \in F}\right)<\left(\varepsilon+\frac{1}{l}\right)\left|F_{k}\right|
$$

for some $k \geq m$ is open, and by the continuity properties of entropy $S_{\varepsilon}$ is equal to $\bigcap_{l=1}^{\infty} \bigcap_{n=1}^{\infty} \bigcap_{m=1}^{\infty} S_{\varepsilon}(n, m, l)$, which is a $G_{\delta}$. Kawahigashi showed in [30] that there exist free actions realizing all possible nonzero values of entropy in the sense of Connes and Størmer, to which CNT entropy specializes in this case [37. Since the orbit of every free action is dense in $\operatorname{Act}(G, R)$ by Lemma 5.2, we conclude that $S_{\varepsilon}$ is dense.

Remark 5.4. For any von Neumann algebra $M$ with separable predual and faithful normal state $\tau$, the set of $\alpha \in \operatorname{Act}(G, M, \tau)$ with $h_{\tau}(\alpha)=0$ is a $G_{\delta}$. To see this one can argue as in the proof of Lemma 5.3 by taking the sequence $\left\{\gamma_{n}\right\}_{n=1}^{\infty}$ to consist of a union of countable point- $\tau$-norm dense sets of channels $B \rightarrow M$ over a collection of representatives $B$ of the countably many isomorphism classes of finite-dimensional $C^{*}$-algebras.

The notions of joinings and disjointness [19] can be extended to noncommutative actions as follows. A joining is a certain type of correspondence [12, App. V.B] [43] and as such we need to consider opposite algebras in order to formulate the definition as in the commutative case. For a $C^{*}$-algebra $A$ we write $A^{\text {op }}$ for the opposite $C^{*}$-algebra, which has the same *-linear structure as $A$ but with the multiplication reversed. If $A$ is a von Neumann algebra then so is $A^{\text {op }}$. For an element $b \in A$ we write $\tilde{b}$ for the corresponding element of $A^{\text {op }}$. An action $\beta$ on $A$ gives rise to an action $\beta^{\text {op }}$ on $A^{\text {op }}$ defined by $\beta_{s}^{\text {op }}(\tilde{a})=\widetilde{\beta_{s}(a)}$ for all $s \in G$ and $a \in N$.

Definition 5.5. Let $M$ and $N$ be von Neumann algebras with faithful normal tracial states $\tau$ and $\sigma$, respectively. Let $G$ a locally compact group. Let $\alpha \in \operatorname{Act}(M, G, \tau)$ and $\beta \in \operatorname{Act}(N, G, \sigma)$. A joining of $\alpha$ and $\beta$ is an $\left(\alpha \otimes \beta^{\text {op }}\right)$-invariant state on the maximal $C^{*}$-tensor product $M \otimes_{\max } N^{\mathrm{op}}$ whose marginals are $\tau$ and $\sigma$. We say that $\alpha$ and $\beta$ are disjoint if $\tau \otimes \sigma$ is the only joining of $\alpha$ and $\beta$.

Note that the definition of joining is symmetric in the sense that an $\left(\alpha \otimes \beta^{\mathrm{op}}\right)$-invariant state on $M \otimes_{\max } N^{\mathrm{op}}$ corresponds to an $\left(\beta \otimes \alpha^{\mathrm{op}}\right)$-invariant state on $N \otimes_{\max } M^{\mathrm{op}}$ via the canonical isomorphism of the latter with the opposite $C^{*}$-algebra of $M \otimes_{\max } N^{\mathrm{op}}$.

Specializing the picture for general correspondences between von Neumann algebras [12, App. V.B] [43, \$1.2], we see that there is a bijective correspondence between the joinings of two actions $\alpha \in \operatorname{Act}(M, G, \tau)$ and $\beta \in \operatorname{Act}(N, G, \sigma)$ and the $G$-equivariant unital complete positive maps $\varphi: M \rightarrow N$ such that $\tau=\sigma \circ \varphi$. We associate to a joining $\omega$ of $\alpha$ with $\beta$ such a unital completely positive $\operatorname{map} \varphi: M \rightarrow N$ as follows. We define the bounded operator $S: L^{2}(N, \sigma) \rightarrow L^{2}\left(M \otimes_{\max } N^{\mathrm{op}}, \omega\right)$ by setting $S a=\mathbf{1}_{M} \otimes \tilde{a}$ for $a \in N$, viewing all of these elements as vectors in the appropriate GNS Hilbert space. We also define the representation $\pi: M \rightarrow \mathcal{B}\left(L^{2}\left(M \otimes_{\max } N^{\mathrm{op}}, \omega\right)\right)$ by $\pi(a) \zeta=\left(a \otimes \mathbf{1}_{N^{\mathrm{op}}}\right) \zeta$, viewing $M \otimes \mathbf{1}_{N^{\mathrm{op}}}$ as acting on $L^{2}\left(M \otimes_{\max } N^{\mathrm{op}}, \omega\right)$. For $a \in M$ set $\varphi(a)=S^{*} \pi(a) S$. Then $S^{*} \pi(a) S$ commutes with right multiplication by elements of $N$ on $L^{2}(N, \sigma)$, so that $\varphi(a) \in\left(N^{\prime}\right)^{\prime}=N$. 
In the reverse direction, given a $G$-equivariant unital complete positive map $\varphi: M \rightarrow N$ such that $\tau=\sigma \circ \varphi$, we define on $\mathcal{H}_{0}=M \otimes N$ the sesquilinear form

$$
\left\langle a_{1} \otimes b_{1}, a_{2} \otimes b_{2}\right\rangle_{\varphi}=\sigma\left(\varphi\left(a_{2}^{*} a_{1}\right) b_{1} b_{2}^{*}\right),
$$

take the completion $\mathcal{H}$ of $\mathcal{H}_{0}$ modulo the null space of $\langle\cdot, \cdot\rangle_{\varphi}$, and observe that the left and right actions of $M$ and $N$, respectively, pass to commuting actions on $\mathcal{H}$. This gives a representation of $M \otimes_{\max } N^{\mathrm{op}}$, with $G$-invariant vector state $\omega$ arising from the class of $\mathbf{1}_{M} \otimes \mathbf{1}_{N}$ in $\mathcal{H}$ and having $\tau$ and $\sigma$ as marginals. Note in particular that the identity map on $M$ gives a joining of $\alpha$ with itself (the diagonal joining) which, as long as $M \neq \mathbb{C}$, is different from the product joining. Thus every action in $\operatorname{Act}(G, M, \tau)$ for $M \neq \mathbb{C}$ is not disjoint from any of its conjugates.

It is easily checked that, under the above correspondence, the image of $\varphi$ is the scalars precisely when it corresponds to the product state $\tau \otimes \sigma$ by the assumption on the marginals in the definition of joining. This observation was used in 34 to a give a linear-geometric proof of the disjointness of zero entropy and completely positive entropy actions of discrete amenable groups on a probability space. We will also apply this perspective in the proof of the following lemma, only now using CNT entropy instead of $\ell_{1}$ geometry.

Lemma 5.6. Suppose that $G$ is countably infinite and amenable. Let $\alpha$ be an action in $\operatorname{Act}(G, R)$ with $h_{\tau}(\alpha)=0$. Then $\alpha$ is disjoint from every action in $\operatorname{Act}(G, R)$ which is conjugate to the Bernoulli shift on the weak operator closure of $M_{2}^{\otimes G}$.

Proof. We view $R$ as $\left(M_{2}, \operatorname{tr}\right)^{\otimes G}$ and write $\beta$ for the Bernoulli shift on the latter. Let $\gamma: B \rightarrow R$ be a channel. By Proposition 3.1.11 of [37] we can find a channel $\gamma^{\prime}: B \rightarrow R$ such that $\left|H_{\tau}\left(\gamma^{\prime}\right)-H_{\tau}(\gamma)\right| \leq H_{\tau}(\gamma) / 4$. and $\gamma^{\prime}(B) \subseteq M_{2}^{\otimes K}$ for some finite set $K \subseteq G$. Given a nonempty finite set $F \subseteq G$, take a subset $F^{\prime} \subseteq F$ which is maximal with respect to the property that $s \notin K K^{-1} t$ for all distinct $s, t \in F^{\prime}$. Then $\left|F^{\prime}\right| \geq|F| /\left|K K^{-1}\right|$ and $H_{\tau}\left(\left(\beta_{s} \circ \gamma^{\prime}\right)_{s \in F^{\prime}}\right)=\left|F^{\prime}\right| H_{\tau}\left(\gamma^{\prime}\right)$, and hence

$$
\begin{aligned}
H_{\tau}\left(\left(\beta_{s} \circ \gamma\right)_{s \in F^{\prime}}\right) & \geq H_{\tau}\left(\left(\beta_{s} \circ \gamma^{\prime}\right)_{s \in F^{\prime}}\right)-\frac{1}{4}\left|F^{\prime}\right| H_{\tau}(\gamma) \\
& =\left|F^{\prime}\right|\left(H_{\tau}\left(\gamma^{\prime}\right)-\frac{1}{4} H_{\tau}(\gamma)\right) \\
& \geq \frac{1}{2}\left|F^{\prime}\right| H_{\tau}(\gamma)
\end{aligned}
$$

Now suppose we are given a $\tau$-preserving u.c.p. map $\varphi: R \rightarrow R$ such that $\varphi \circ \alpha_{s}=\beta_{s} \circ \varphi$ for all $s \in G$. Then applying the above inequality to $\varphi \circ \gamma$ and using the monotonicity properties of $H_{\tau}(\cdot)$ we have

$$
\begin{aligned}
\frac{1}{2}\left|F^{\prime}\right| H_{\tau}(\varphi \circ \gamma) & \leq H_{\tau}\left(\left(\beta_{s} \circ \varphi \circ \gamma\right)_{s \in F^{\prime}}\right) \\
& =H_{\tau}\left(\left(\varphi \circ \alpha_{s} \circ \gamma\right)_{s \in F^{\prime}}\right) \\
& \leq H_{\tau}\left(\left(\alpha_{s} \circ \gamma\right)_{s \in F^{\prime}}\right)
\end{aligned}
$$

and so

$$
\frac{1}{|F|} H_{\tau}\left(\left(\alpha_{s} \circ \gamma\right)_{s \in F}\right) \geq \frac{1}{\left|F^{\prime}\right|\left|K K^{-1}\right|} H_{\tau}\left(\left(\alpha_{s} \circ \gamma\right)_{s \in F^{\prime}}\right)
$$




$$
\geq \frac{1}{2\left|K K^{-1}\right|} H_{\tau}(\varphi \circ \gamma)
$$

But $\frac{1}{|F|} H_{\tau}\left(\left(\alpha_{s} \circ \gamma\right)_{s \in F}\right) \rightarrow 0$ as $F$ becomes more and more invariant since $\alpha$ has zero entropy. Therefore $H_{\tau}(\varphi \circ \gamma)=0$, and so $\varphi \circ \gamma$ maps into the scalars [37, Lemma 3.1.4]. It follows that $\varphi$ is the map $a \mapsto \tau(a) \mathbf{1}_{R}$, yielding the lemma.

The following generalizes a result of del Junco from the commutative case [14].

Lemma 5.7. Let $M$ be a von Neumann algebra with separable predual and faithful normal tracial state $\tau$. Let $\alpha \in \operatorname{Act}(G, M, \tau)$. Then the set $\alpha^{\perp}$ of actions in $\operatorname{Act}(G, M, \tau)$ that are disjoint from $\alpha$ is $a G_{\delta}$.

Proof. For a compact set $K \subseteq G$, finite sets $\Omega, \Theta \subseteq M$ and $\Upsilon \subseteq M_{*}$, and $\varepsilon>0$ we write $S(K, \Omega, \Theta, \Upsilon, \varepsilon)$ for the set of all $\beta \in \operatorname{Act}(G, M, \tau)$ for which there exists a $\delta>0$ such that if $\varphi: M \rightarrow M$ is a $\tau$-preserving u.c.p. map satisfying $\left\|\alpha_{s}(\varphi(a))-\varphi\left(\beta_{s}(a)\right)\right\|_{2}<\delta$ for all $s \in K$ and $a \in \Omega$ then $|\omega(\varphi(a))-\tau(a) \omega(\mathbf{1})|<\varepsilon$ for all $a \in \Theta$ and $\omega \in \Upsilon$. It is readily checked that $S(K, \Omega, \Theta, \Upsilon, \varepsilon)$ is open. Take an increasing sequence $K_{1} \subseteq K_{2} \subseteq \cdots$ of compact subsets of $G$ such that $\bigcup_{n=1}^{\infty} K_{n}$ is dense in $G$, and increasing sequences $\Omega_{1} \subseteq \Omega_{2} \subseteq \cdots$ and $\Upsilon_{1} \subseteq$ $\Upsilon_{2} \subseteq \cdots$ of finite subsets of $M$ and $M_{*}$, respectively, such that $\bigcup_{n=1}^{\infty} \Omega_{n}$ is $\tau$-norm dense in $M$ and $\bigcup_{n=1}^{\infty} \Upsilon_{n}$ is norm dense in $M_{*}$. Then $S:=\bigcap_{n=1}^{\infty} \bigcup_{m=1}^{\infty} S\left(K_{m}, \Omega_{m}, \Omega_{n}, \Upsilon_{n}, 1 / n\right)$ is a $G_{\delta}$, and to complete the proof we will show that it is equal to $\alpha^{\perp}$.

Clearly $S \subseteq \alpha^{\perp}$. So let $\beta \in \operatorname{Act}(G, M, \tau) \backslash S$ and let us show that $\beta \notin \alpha^{\perp}$. For some $n \in \mathbb{N}$ we have $\beta \notin \bigcup_{m=1}^{\infty} S\left(K_{m}, \Omega_{m}, \Omega_{n}, \Upsilon_{n}, 1 / n\right)$. Then for every $m \in \mathbb{N}$ there is a $\tau$-preserving u.c.p. map $\varphi_{m}: M \rightarrow M$ such that $\left\|\alpha_{s}\left(\varphi_{m}(a)\right)-\varphi_{m}\left(\beta_{s}(a)\right)\right\|_{2}<1 / m$ for all $s \in K_{m}$ and $a \in \Omega_{m}$ and $\sup _{a \in \Omega_{n}, \omega \in \Upsilon_{n}}\left|\omega\left(\varphi_{m}(a)\right)-\tau(a) \omega(\mathbf{1})\right| \geq 1 / n$. We can then find a subsequence $\left\{\varphi_{m_{k}}\right\}_{k=1}^{\infty}$, an $a_{0} \in \Omega_{n}$, and an $\omega_{0} \in \Upsilon_{n}$ such that $\mid \omega_{0}\left(\varphi_{m_{k}}\left(a_{0}\right)\right)$ $\tau\left(a_{0}\right) \omega_{0}(\mathbf{1}) \mid \geq 1 / n$ for all $k$. Now take a point-weak* limit point $\varphi$ of $\left\{\varphi_{m_{k}}\right\}_{k=1}^{\infty}$. Then $\varphi$ is $\tau$-preserving, $\alpha_{s} \circ \varphi=\varphi \circ \beta_{s}$ for all $s \in G$, and $\left|\omega_{0}\left(\varphi\left(a_{0}\right)\right)-\tau\left(a_{0}\right) \omega_{0}(\mathbf{1})\right| \geq 1 / n$, so that $\varphi$ defines a joining different from the product one. Thus $\beta \notin \alpha^{\perp}$, and so $S=\alpha^{\perp}$, as desired.

Lemma 5.8. Suppose that $G$ is countably infinite and amenable. Let $\alpha$ be an action in $\operatorname{Aut}(G, R)$ with $h_{\tau}(\alpha)=0$. Then $\alpha^{\perp}$ is a dense $G_{\delta}$ subset of $\operatorname{Aut}(G, R)$.

Proof. By Lemma 5.7 it suffices to show that $\alpha^{\perp}$ is dense. As observed in Subsection 4.1 . the Bernoulli shift on the tracial weak operator closure of $M_{2}^{\otimes G}$ is free, and so any action in $\operatorname{Act}(G, R)$ that is conjugate to it has dense orbit by Lemma 5.2. Thus $\alpha^{\perp}$ is dense by Lemma 5.6.

Lemma 5.9. Suppose that $G$ is countably infinite and amenable. Then every orbit in $\operatorname{Act}(G, R)$ is meager.

Proof. Let $\alpha \in \operatorname{Act}(G, R)$ and suppose that the orbit is not meager. Then $h_{\tau}(\alpha)=0$ by Lemma 5.3. But then $\alpha^{\perp}$ is a dense $G_{\delta}$ by 5.8 and every action in $\alpha^{\perp}$ is disjoint from every action in the orbit of $\alpha$, yielding a contradiction.

5.2. Turbulence. All infinite tensor products in what follows are with respect to the trace. 
Lemma 5.10. Let $\left\{\alpha_{i}\right\}_{i=1}^{\infty}$ be a dense sequence in $\operatorname{Act}(G, R)$, and let $\alpha$ be an element of $\operatorname{Act}(G, R)$ which is conjugate to $\bigotimes_{i=1}^{\infty}\left(\alpha_{i} \otimes \operatorname{id}_{R}\right) \in \operatorname{Act}\left(G,(R \bar{\otimes} R)^{\bar{\otimes} \mathbb{N}}\right)$. Then $\alpha$ is a turbulent point and has dense orbit for the action of $\operatorname{Aut}(R)$ on $\operatorname{Act}(G, R)$.

Proof. To show that $\alpha$ is a turbulent point, let $Y$ be a neighbourhood of $\alpha$ in $\operatorname{Act}(G, R)$ and $Z$ a neighbourhood of $\operatorname{id}_{R}$ in $\operatorname{Aut}(R)$ and let us demonstrate that the closure of the local orbit $\mathcal{O}(\alpha, Y, Z)$ has nonempty interior. By shrinking $Y$ and $Z$ if necessary we may suppose that $Y=Y_{\alpha, K, \Omega, \varepsilon}$ and $Z=Z_{\mathrm{id}, \Omega, \varepsilon}$ for some compact set $K \subseteq G$, finite set $\Omega \subseteq R$, and $\varepsilon>0$.

Let $\theta \in Y$. We will argue that $\theta$ is contained in $\overline{\mathcal{O}(\alpha, Y, Z)}$. Let $L$ be a compact subset of $G$ containing $K$ and $\Upsilon$ a finite subset of $R$ containing $\Omega$. Let $\delta>0$ be such that $\left\|\theta_{s}(a)-\alpha_{s}(a)\right\|_{2}<\varepsilon-\delta$ for all $a \in \Omega$ and $s \in K$. For simplicity we will view $R$ as $(R \bar{\otimes} R)^{\bar{\otimes} \mathbb{N}}$ with $\alpha$ acting as $\bigotimes_{i=1}^{\infty}\left(\alpha_{i} \otimes \mathrm{id}_{R}\right)$. Take a $k \in \mathbb{N}$ large enough so that there is a finite-dimensional subfactor $N \subseteq(R \bar{\otimes} R)^{\bar{\otimes}[1, k]}$ such that for every $a \in \bigcup_{s \in L \cup\{e\}} \theta_{s}(\Upsilon)$ we have $\|E(a)-a\|_{2}<\delta / 12$ where $E$ is the trace-preserving conditional expectation from $(R \bar{\otimes} R)^{\bar{\otimes} \mathbb{N}}$ onto $N \otimes \mathbf{1}$, with $\mathbf{1}$ denoting here and for the remainder of the paragraph the unit in $(R \bar{\otimes} R)^{\bar{\otimes}[k+1, \infty]}$. For $a \in \Upsilon$ we write $a^{\prime}$ for the element of $N$ such that $E(a)=a^{\prime} \otimes \mathbf{1}$. Extend the embedding $N \hookrightarrow(R \bar{\otimes} R)^{\bar{\otimes} \mathbb{N}}$ given by $a \mapsto a \otimes \mathbf{1}$ to an isomorphism $\Phi$ : $(R \bar{\otimes} R)^{\bar{\otimes}[1, k]} \rightarrow(R \bar{\otimes} R)^{\bar{\otimes} \mathbb{N}}$ and define the action $\tilde{\theta}$ of $G$ on $(R \bar{\otimes} R)^{\bar{\otimes}[1, k]}$ by $s \mapsto \Phi^{-1} \circ \theta_{s} \circ \Phi$. For $a \in \Upsilon$ and $s \in L$ we have, noting that $\Phi\left(a^{\prime}\right)=E(a)$ and $\Phi^{-1}\left(E\left(\theta_{s}(a)\right)\right) \otimes \mathbf{1}=E\left(\theta_{s}(a)\right)$,

$$
\begin{aligned}
\left\|\theta_{s}(a)-\tilde{\theta}_{s}\left(a^{\prime}\right) \otimes \mathbf{1}\right\|_{2} & =\left\|\theta_{s}(a)-\Phi^{-1}\left(\theta_{s}(E(a))\right) \otimes \mathbf{1}\right\|_{2} \\
\leq & \left\|\theta_{s}(a)-E\left(\theta_{s}(a)\right)\right\|_{2}+\left\|\Phi^{-1}\left(E\left(\theta_{s}(a)\right)-\theta_{s}(a)\right) \otimes \mathbf{1}\right\|_{2} \\
& \quad+\left\|\Phi^{-1}\left(\theta_{s}(a-E(a))\right) \otimes \mathbf{1}\right\|_{2} \\
& <\frac{\delta}{12}+\frac{\delta}{12}+\frac{\delta}{12}=\frac{\delta}{4} .
\end{aligned}
$$

Abbreviate $\bigotimes_{i=1}^{k}\left(\alpha_{i} \otimes \mathrm{id}_{R}\right)$ to $\sigma$ and fix an identification of $(R \bar{\otimes} R)^{\bar{\otimes}[1, k]}$ with $R$, so that $\sigma$ and $\tilde{\theta}$ are regarded as actions on $R$. By the density of the sequence $\left\{\alpha_{i}\right\}_{i=1}^{\infty}$ we can find an integer $l>k$ such that $\left\|\tilde{\theta}_{s}\left(a^{\prime}\right)-\alpha_{l, s}\left(a^{\prime}\right)\right\|_{2}<\delta / 4$ for all $s \in L$ and $a \in \Upsilon$. Note that, for all $s \in K$ and $a \in \Omega$,

$$
\begin{aligned}
\left\|\sigma_{s}\left(a^{\prime}\right)-\alpha_{l, s}\left(a^{\prime}\right)\right\|_{2} & \leq\left\|\sigma_{s}\left(a^{\prime}\right) \otimes \mathbf{1}-\alpha_{s}(a)\right\|_{2}+\left\|\alpha_{s}(a)-\theta_{s}(a)\right\|_{2} \\
& \quad+\left\|\theta_{s}(a)-\tilde{\theta}_{s}\left(a^{\prime}\right) \otimes \mathbf{1}\right\|_{2}+\left\|\tilde{\theta}_{s}\left(a^{\prime}\right)-\alpha_{l, s}\left(a^{\prime}\right)\right\|_{2} \\
& <\frac{\delta}{12}+\varepsilon-\delta+\frac{\delta}{4}+\frac{\delta}{4} \\
& =\varepsilon-\frac{5 \delta}{12} .
\end{aligned}
$$

Take an integer $n>64 \varepsilon^{-2} \max \{\|a\|: a \in \Omega\}$. Since $R \cong M_{n} \otimes R$ and $\alpha$ contains infinitely many tensor product factors equal to id $R$, we can view $\alpha$ as $\sigma \otimes \alpha_{l} \otimes \operatorname{id}_{N} \otimes \rho$ acting on $R \bar{\otimes} R \bar{\otimes} N \bar{\otimes} R$ where $N$ is a $\mathrm{I}_{n}$ factor and $\rho$ is some automorphism of $R$. Let $C$ be a commutative $n$-dimensional ${ }^{*}$-subalgebra of $N$. Let $e_{1}, \ldots, e_{n}$ be the minimal projections of $C$. For a set $E \subseteq\{1, \ldots, n\}$ we write $\gamma_{E}$ for the automorphism of $R \bar{\otimes} R \otimes C$ which 
sends $\sum_{i=1}^{n} a_{i} \otimes e_{i} \in(R \bar{\otimes} R) \otimes C$ to

$$
\sum_{i \in E} \beta\left(a_{i}\right) \otimes e_{i}+\sum_{i \in\{1, \ldots, n\} \backslash E} a_{i} \otimes e_{i}
$$

where $\beta$ is the tensor product flip automorphism of $R \bar{\otimes} R$. Then for every $a \in \Upsilon$ and $s \in L$ the image $a^{\prime \prime}$ of $a^{\prime} \otimes \mathbf{1}_{R \otimes C}$ under $\gamma_{E} \cdot\left(\sigma_{s} \otimes \alpha_{l, s} \otimes \mathrm{id}_{C}\right)$ is equal to

$$
\sigma_{s}\left(a^{\prime}\right) \otimes \mathbf{1}_{R} \otimes(1-p)+\alpha_{l, s}\left(a^{\prime}\right) \otimes \mathbf{1}_{R} \otimes p
$$

where $p$ is the projection $\sum_{i \in E} e_{i}$, and hence, for $a \in \Omega$ and $s \in K$,

$$
\left\|a^{\prime \prime}-\sigma_{s}\left(a^{\prime}\right) \otimes \mathbf{1}_{R \otimes C}\right\|_{2} \leq\left\|\sigma_{s}\left(a^{\prime}\right)-\alpha_{l, s}\left(a^{\prime}\right)\right\|_{2}\|p\|_{2}<\varepsilon-\frac{5 \delta}{12} .
$$

Since every automorphism of the hyperfinite $\mathrm{II}_{1}$ factor is approximately inner [46, Thm. 2.16] and $\gamma_{E}$ fixes the elements of $\mathbf{1}_{R \bar{\otimes} R} \otimes C$, we can find a unitary $u \in R \bar{\otimes} R \otimes C$ such that $\left\|u b u^{*}-\gamma_{E}(b)\right\|_{2}<\delta / 8$ for all elements $b$ equal to $\left(\left(\sigma_{s} \otimes \alpha_{l, s} \otimes \mathrm{id}_{C}\right) \circ \gamma_{E}^{-1}\right)\left(a^{\prime} \otimes \mathbf{1}_{R \otimes C}\right)$ for some $s \in L \cup\{e\}$ and $a \in \Upsilon$ or to $a^{\prime} \otimes \mathbf{1}_{R \otimes C}$ for some $a \in \Upsilon$. Regarding $R \bar{\otimes} R \otimes C$ henceforth as a subalgebra of $R \bar{\otimes} R \bar{\otimes} N \bar{\otimes} R$ under the embedding given on elementary tensors by $a \otimes a \otimes c \mapsto a \otimes a \otimes c \otimes \mathbf{1}_{R}$, we set $\hat{\gamma}_{E}=\operatorname{Ad} u \in \operatorname{Aut}(R \bar{\otimes} R \bar{\otimes} N \bar{\otimes} R)$. With an untagged 1 denoting henceforth the unit of $R \bar{\otimes} N \bar{\otimes} R$, for all $a \in \Upsilon$ we have, noting that $\gamma_{E}^{-1}\left(a^{\prime} \otimes \mathbf{1}\right)$ makes sense as $a^{\prime} \otimes \mathbf{1}$ lies in the subalgebra of which $\gamma_{E}$ is an automorphism,

$$
\left\|\hat{\gamma}_{E}^{-1}\left(a^{\prime} \otimes \mathbf{1}\right)-\gamma_{E}^{-1}\left(a^{\prime} \otimes \mathbf{1}\right)\right\|_{2}=\left\|\gamma_{E}\left(\gamma_{E}^{-1}\left(a^{\prime} \otimes \mathbf{1}\right)\right)-\hat{\gamma}_{E}\left(\gamma_{E}^{-1}\left(a^{\prime} \otimes \mathbf{1}\right)\right)\right\|_{2}<\frac{\delta}{8}
$$

and hence, writing $\tilde{\alpha}$ for the restriction $s \mapsto \sigma_{s} \otimes \alpha_{l, s} \otimes \operatorname{id}_{C}$ of $\alpha$ to $R \bar{\otimes} R \otimes C$, for $s \in L$ we have

$$
\begin{aligned}
\|\left(\hat{\gamma}_{E} \cdot \alpha\right)_{s}\left(a^{\prime} \otimes \mathbf{1}\right) & -\left(\gamma_{E} \cdot \tilde{\alpha}\right)_{s}\left(a^{\prime} \otimes \mathbf{1}\right) \|_{2} \\
\leq & \left\|\hat{\gamma}_{E} \circ \alpha_{s}\left(\hat{\gamma}_{E}^{-1}\left(a^{\prime} \otimes \mathbf{1}\right)-\gamma_{E}^{-1}\left(a^{\prime} \otimes \mathbf{1}\right)\right)\right\|_{2} \\
& \quad+\| \hat{\gamma}_{E}\left(\alpha_{s} \circ \gamma_{E}^{-1}\left(a^{\prime} \otimes \mathbf{1}\right)\right)-\gamma_{E}\left(\alpha_{s} \circ \gamma_{E}^{-1}\left(a^{\prime} \otimes \mathbf{1}\right) \|_{2}\right. \\
& <\frac{\delta}{4} .
\end{aligned}
$$

Thus for $a \in \Omega$ and $s \in K$ we have

$$
\begin{aligned}
\left(\hat{\gamma}_{E} \cdot \alpha\right)_{s}(a) & \approx_{\delta / 12}\left(\hat{\gamma}_{E} \cdot \alpha\right)_{s}\left(a^{\prime} \otimes \mathbf{1}\right) \\
& \approx_{\delta / 4}\left(\gamma_{E} \cdot \tilde{\alpha}\right)_{s}\left(a^{\prime} \otimes \mathbf{1}\right) \\
& \approx_{\varepsilon-5 \delta / 12} \sigma_{s}\left(a^{\prime}\right) \otimes \mathbf{1} \\
& =\alpha_{s}\left(a^{\prime} \otimes \mathbf{1}\right) \\
& \approx_{\delta / 12} \alpha_{s}(a)
\end{aligned}
$$

so that $\hat{\gamma}_{E} \cdot \alpha \in Y$, while in the case that $E=\{1, \ldots, n\}$ we have, for $a \in \Upsilon$ and $s \in L$,

$$
\begin{aligned}
\left(\hat{\gamma}_{E} \cdot \alpha\right)_{s}(a) & \approx_{\delta / 12}\left(\hat{\gamma}_{E} \cdot \alpha\right)_{s}\left(a^{\prime} \otimes \mathbf{1}\right) \\
& \approx_{\delta / 4}\left(\gamma_{\{1, \ldots, n\}} \cdot \tilde{\alpha}\right)_{s}\left(a^{\prime} \otimes \mathbf{1}\right) \\
& =\alpha_{l, s}\left(a^{\prime}\right) \otimes \mathbf{1} \\
& \approx_{\delta / 4} \tilde{\theta}_{s}\left(a^{\prime}\right) \otimes \mathbf{1}
\end{aligned}
$$




$$
\approx_{\delta / 4} \theta_{s}(a)
$$

so that $\hat{\gamma}_{E} \cdot \alpha \in Y_{\theta, L, \Upsilon, \delta}$.

Note that, for all $i=1, \ldots, n$ and $a \in \Omega$,

$$
\left\|\gamma_{\{i\}}\left(a^{\prime} \otimes \mathbf{1}\right)-a^{\prime} \otimes \mathbf{1}\right\|_{2} \leq\left\|\beta\left(a^{\prime}\right)-a^{\prime}\right\|_{2}\left\|e_{i}\right\|_{2}<\frac{2\|a\|}{\sqrt{n}}
$$

and thus

$$
\begin{aligned}
\left\|\hat{\gamma}_{\{i\}}(a)-a\right\|_{2} \leq & \left\|\hat{\gamma}_{\{i\}}\left(a-a^{\prime} \otimes \mathbf{1}\right)\right\|_{2}+\left\|\hat{\gamma}_{\{i\}}\left(a^{\prime} \otimes \mathbf{1}\right)-\gamma_{\{i\}}\left(a^{\prime} \otimes \mathbf{1}\right)\right\|_{2} \\
& +\left\|\gamma_{\{i\}}\left(a^{\prime} \otimes \mathbf{1}\right)-a^{\prime} \otimes \mathbf{1}\right\|_{2}+\left\|a^{\prime} \otimes \mathbf{1}-a\right\|_{2} \\
& <\frac{\varepsilon}{4}+\frac{\varepsilon}{4}+\frac{2\|a\|}{\sqrt{n}}+\frac{\varepsilon}{4} \\
& <\varepsilon,
\end{aligned}
$$

so that $\hat{\gamma}_{\{i\}} \in Z$. From the previous paragraph, the action $\left(\hat{\gamma}_{\{j\}} \circ \hat{\gamma}_{\{j-1\}} \circ \cdots \circ \hat{\gamma}_{\{1\}}\right) \cdot \alpha=$ $\hat{\gamma}_{\{1, \ldots, j\}} \cdot \alpha$ is contained in $Y$ for every $j=1, \ldots, n$, while $\left(\hat{\gamma}_{\{n\}} \circ \hat{\gamma}_{\{n-1\}} \circ \cdots \circ \hat{\gamma}_{\{1\}}\right) \cdot \alpha=$ $\hat{\gamma}_{\{1, \ldots, n\}} \cdot \alpha$ is contained in $Y_{\theta, L, \Upsilon, \delta}$. We conclude that $\theta \in \overline{\mathcal{O}(\alpha, Y, Z)}$ and hence that $Y \subseteq \overline{\mathcal{O}(\alpha, Y, Z)}$, so that $\overline{\mathcal{O}(\alpha, Y, Z)}$ has nonempty interior, as desired.

The density of the orbit of $\alpha$ now also follows, since we showed that $Y \subseteq \overline{\mathcal{O}(\alpha, Y, Z)}$ for any $Y$ of the form $Y_{\alpha, K, \Omega, \varepsilon}$.

The following is the analogue of Theorem 13.3 in [31].

Theorem 5.11. The following conditions are equivalent:

(1) every $\operatorname{Aut}(R)$-orbit in $\operatorname{Act}(G, R)$ is meager,

(2) the action of $\operatorname{Aut}(R)$ on $\operatorname{Act}(G, R)$ is generically turbulent.

Moreover if $G$ does not have property $T$ then (1) and (2) are equivalent to each of the following:

(3) every $\operatorname{Aut}(R)$-orbit in $\mathrm{WM}(G, R)$ is meager,

(4) the action of $\operatorname{Aut}(R)$ on $\operatorname{WM}(G, R)$ is generically turbulent.

Proof. By [35, Thm. 3.8], the $G_{\delta}$ subset $\operatorname{WM}(G, R)$ of $\operatorname{Act}(G, R)$ is dense precisely when $G$ does not have property T. Thus, since meagerness of orbits is part of the definition of generic turbulence, it suffices to show $(1) \Rightarrow(2)$, and this follows by Lemma 5.10 .

Remark 5.12. By Lemma 5.1, when $G$ is countable and does not have property $\mathrm{T}$ we can replace $\operatorname{WM}(G, R)$ by the set of free weakly mixing actions in $\operatorname{Act}(G, R)$ in the above theorem.

Lemma 5.13. Suppose that $G$ is countable and amenable. Let $\alpha$ be a free action of $G$ on $R$. Then $\alpha$ is a turbulent point for the action of $\operatorname{Aut}(R)$ on $\operatorname{Act}(G, R)$.

Proof. By Lemma 5.10 there exists an action $\beta$ of $G$ on $R$ such that for any finite set $K \subseteq G$ and $\Omega \subseteq R$ and $\varepsilon>0$, the neighbourhood $Y=Y_{\beta, K, \Omega, \varepsilon}$ of $\beta$ is contained in $\overline{\mathcal{O}(\beta, Y, Z)}$, where $Z=Z_{\mathrm{id}, \Omega, \varepsilon}$. Note that $G$ has a free action on $R$ by Lemma 5.1 , and so we may assume that $\beta$ is free by taking $\alpha_{1}$ in Lemma 5.10 to be free, since the tensor product of a free action with any other action is free [29, Cor. 1.12]. For 
a $\gamma \in \operatorname{Aut}(R)$ we have $\gamma Y_{\beta, K, \Omega, \varepsilon}=Y_{\gamma \cdot \beta, K, \gamma \Omega, \varepsilon}$ and $\gamma Z_{\mathrm{id}, \Omega, \varepsilon} \gamma^{-1}=Z_{\mathrm{id}, \gamma \Omega, \varepsilon}$, and hence $\gamma \mathcal{O}\left(\beta, Y_{\beta, K, \Omega, \varepsilon}, Z_{\mathrm{id}, \Omega, \varepsilon}\right)=\mathcal{O}\left(\gamma \cdot \beta, Y_{\gamma \cdot \beta, K, \gamma \Omega, \varepsilon}, Z_{\mathrm{id}, \gamma \Omega, \varepsilon}\right)$. Thus $\gamma \cdot \beta$ also has the property according to which $\beta$ was chosen.

Now let $Y$ and $Z$ be neighbourhoods of $\alpha$ and $\operatorname{id}_{R}$ in $\operatorname{Act}(G, R)$ and $\operatorname{Aut}(R)$, respectively. Shrinking $Y$ and $Z$ if necessary, we may assume that $Y=Y_{\alpha, K, \Omega, \varepsilon}$ and $Z=Z_{\mathrm{id}, \Omega, \varepsilon}$ for some $K, \Omega$, and $\varepsilon$ as above. We claim that $Y \subseteq \overline{\mathcal{O}(\alpha, Y, Z)}$. Let $\theta \in Y$. Then $\theta \in Y_{\alpha, K, \Omega, \varepsilon^{\prime}}$ for some $0<\varepsilon^{\prime}<\varepsilon$. Let $L$ be a finite subset of $G$ containing $K, \Upsilon$ a finite subset of $R$ containing $\Omega$, and $\delta \in\left(0,\left(\varepsilon-\varepsilon^{\prime}\right) / 2\right)$. By the theorem of Ocneanu in Section 1.4 of [38], we can find unitaries $u_{s} \in R$ for $s \in L$ and $\gamma \in \operatorname{Aut}(R)$ such that $\left\|u_{s}-1\right\|_{2}<\delta / 4$ and $(\gamma \cdot \beta)_{s}=\operatorname{Ad} u_{s} \circ \alpha_{s}$ for all $s \in L$. Note that

$$
\begin{aligned}
\left\|(\rho \cdot(\gamma \cdot \beta))_{s}(a)-(\rho \cdot \alpha)_{s}(a)\right\|_{2} & =\left\|\rho\left(u_{s}\right)\left((\rho \cdot \alpha)_{s}(a)\right) \rho\left(u_{s}\right)^{*}-(\rho \cdot \alpha)_{s}(a)\right\|_{2} \\
& \leq 2\left\|\rho\left(u_{s}\right)-1\right\|_{2}=2\left\|u_{s}-1\right\|_{2} \\
& <\delta / 2
\end{aligned}
$$

for all $s \in L, a \in \Upsilon$ and $\rho \in \operatorname{Aut}(R)$. Set $Y_{\delta}=Y_{\gamma \cdot \beta, K, \Omega, \varepsilon^{\prime}+\delta / 2}$. Then $\theta \in Y_{\delta} \subseteq$ $\overline{\mathcal{O}\left(\gamma \cdot \beta, Y_{\delta}, Z\right)}$. Thus there exists an $\eta \in \mathcal{O}\left(\gamma \cdot \beta, Y_{\delta}, Z\right) \cap Y_{\theta, L, \Upsilon, \delta / 2}$. The above inequality shows that we can find a $\zeta \in \mathcal{O}(\alpha, Y, Z) \cap Y_{\eta, L, \Upsilon, \delta / 2}$. Then $\zeta \in \mathcal{O}(\alpha, Y, Z) \cap Y_{\theta, L, \Upsilon, \delta}$. This proves the claim. Therefore $\alpha$ is a turbulent point.

Theorem 5.14. Suppose that $G$ is countably infinite and amenable. Then the action of $\operatorname{Aut}(R)$ on $\operatorname{Fr}(G, R)$ is turbulent.

Proof. Combine Lemmas 5.1, 5.2, 5.9, and 5.13.

As a consequence of Theorem 3.21 and Corollary 3.19 in [26] and Theorem [5.14, we obtain the following.

Theorem 5.15. Suppose that $G$ is countably infinite and amenable. Then no Aut $(R)$ invariant dense $G_{\delta}$ subset of $\operatorname{Fr}(G, R)$ admits classification by countable structures.

Note that when $G$ does not have property $\mathrm{T}$ the set of weakly mixing actions in $\operatorname{Act}(G, R)$ is a dense $G_{\delta}$ [35, and so when $G$ is countably infinite and amenable the free weakly mixing actions form an $\operatorname{Aut}(R)$-invariant dense $G_{\delta}$ subset of $\operatorname{Fr}(G, R)$ seeing that the latter is a dense $G_{\delta}$ in $\operatorname{Act}(G, R)$ by Lemma 5.1.

\section{Flows And Generic turbulence}

Let $(X, \mu)$ be a standard atomless probability space. The Polish space $\operatorname{Act}(G, X, \mu)$ of continuous $\mu$-preserving actions of a second countable locally compact group $G$ on $(X, \mu)$ with the weak topology can be identified with $\operatorname{Act}\left(G, L^{\infty}(X, \mu), \mu\right)$ as defined in Section 4, The Polish group $\operatorname{Aut}(X, \mu)$ of $\mu$-preserving transformations of $(X, \mu)$ can be viewed as $\operatorname{Act}(\mathbb{Z}, X, \mu)$. We write $\operatorname{Erg}(G, X, \mu)$ for the $G_{\delta}$ subset of ergodic actions in $\operatorname{Act}(G, X, \mu)$. We say that an action $\alpha \in \operatorname{Act}(G, X, \mu)$ is totally ergodic (resp. totally weakly mixing) if the single automorphism $\alpha_{s}$ is ergodic (resp. weakly mixing) for every $s \in G \backslash\{e\}$.

By 45 the set of weakly mixing actions in $\operatorname{Act}(\mathbb{R}, X, \mu)$ is a dense $G_{\delta}$. We have moreover the following.

Lemma 6.1. The set of totally weakly mixing actions in $\operatorname{Act}(\mathbb{R}, X, \mu)$ is comeager. 
Proof. Take an increasing sequence $\mathcal{P}_{1} \leq \mathcal{P}_{2} \leq \ldots$ of finite measurable partitions of $X$ whose union generates a dense subalgebra of the measure algebra. For each $n \in \mathbb{N}$ write $W_{n}$ for the set of all actions $\alpha \in \operatorname{Act}(\mathbb{R}, X, \mu)$ such that there exists a real number $r \geq n$ for which $\left|\mu\left(\alpha_{t}(A) \cap B\right)-\mu(A) \mu(B)\right|<\varepsilon$ for all $A, B \in \mathcal{P}$ and $t \in[r, r+n]$. If we take $\beta$ in the proof of Theorem 4.2 in [35] to be a mixing action (such as the Gaussian action associated to the left regular representation of $\mathbb{R}$ ), then the argument there shows that we can approximate any action in $\operatorname{Act}(\mathbb{R}, X, \mu)$ with one in $W_{n}$. Thus $\bigcap_{n=1}^{\infty} W_{n}$ is a dense $G_{\delta}$, and it consists entirely of totally weakly mixing actions, as is easy to see using the characterization of weak mixing given by Corollary 1.6 of [4].

Theorem 6.2. The action of $\operatorname{Aut}(X, \mu)$ on $\operatorname{Erg}(\mathbb{R}, X, \mu)$ by conjugation is generically turbulent.

Proof. By [45] $\operatorname{Erg}(\mathbb{R}, X, \mu)$ is a dense $G_{\delta}$ in $\operatorname{Act}(\mathbb{R}, X, \mu)$, and so by [26, Thm. 3.21] it suffices to prove that the action of $\operatorname{Aut}(X, \mu)$ on $\operatorname{Act}(\mathbb{R}, X, \mu)$ is generically turbulent. Notice that the proof of Lemma 5.10 works mutatis mutandis with $R$ replaced by $L^{\infty}(X, \mu)$, the main difference being that the approximation of $\gamma_{E}$ by an inner automorphism should be replaced by taking a tensor product of $\gamma_{E}$ with the identity automorphism. Consequently there exists a turbulent point in $\operatorname{Act}(\mathbb{R}, X, \mu)$ with dense orbit. It thus remains to verify that every orbit in $\operatorname{Act}(\mathbb{R}, X, \mu)$ is meager. So let $\alpha \in \operatorname{Act}(\mathbb{R}, X, \mu)$ and let us show that the orbit of $\alpha$ is meager. By Lemma 6.1 we may assume that $\alpha$ is totally ergodic. Note that periodic flows are disjoint from totally ergodic flows, as is easy to see by viewing joinings as unital completely positive maps according to the discussion after Definition 5.5. Since the periodic flows are dense in $\operatorname{Act}(\mathbb{R}, X, \mu)$ by the Rokhlin lemma for flows [40, Lemma 11.1], we deduce in view of Lemma [5.7 that the set of actions in $\operatorname{Act}(\mathbb{R}, X, \mu)$ which are disjoint from $\alpha$ is a dense $G_{\delta}$, so that the orbit of $\alpha$ is meager, as desired.

In the above argument one can also use as a substitute for Lemma 6.1 the fact that the set of flows which are ergodic at nonzero integral times is a dense $G_{\delta}$, which can be deduced from the Rokhlin lemma for flows.

\section{REFERENCES}

[1] L. Baggett. A separable group having a discrete dual space is compact. J. Funct. Anal. 10 (1972), 131-148.

[2] H. Becker and A. S. Kechris. The Descriptive Set Theory of Polish Group Actions. London Mathematical Society Lecture Note Series, 232. Cambridge University Press, Cambridge, 1996.

[3] B. Bekka, P. de la Harpe, and A. Valette. Kazhdan's Property (T). To appear in the New Mathematical Monographs series, Cambridge University Press.

[4] V. Bergelson and J. Rosenblatt. Mixing actions of groups. Ill. J. Math. 32 (1988), 65-80.

[5] B. Blackadar. Operator Algebras. Springer-Verlag, Berlin, 2006.

[6] O. Bratteli and D. W. Robinson. Operator Algebras and Quantum Statistical Mechanics 2. Second edition. Springer-Verlag, Berlin, 1997.

[7] N. P. Brown. On quasidiagonal $C^{*}$-algebras. In: Operator Algebras and Applications, 19-64, Adv. Stud. Pure Math., 38, Math. Soc. Japan, Tokyo, 2004.

[8] J. Bunce and N. Salinas. Completely positive maps on $C^{*}$-algebras and the left essential matricial spectrum of an operator. Duke Math. J. 43 (1976), 747-774.

[9] A. Connes. Outer conjugacy classes of automorphisms of factors. Ann. Sci. École Norm. Sup. (4) 8 (1975), 383-419. 
[10] A. Connes. Periodic automorphisms of the hyperfinite factor of type $\mathrm{II}_{1}$. Acta Sci. Math. (Szeged) 39 (1977), 39-66.

[11] A. Connes, H. Narnhofer, and W. Thirring. Dynamical entropy of $C^{*}$-algebras and von Neumann algebras. Comm. Math. Phs. 112 (1987), 691-719.

[12] A. Connes. Noncommutative Geometry. Academic Press, Inc., San Diego, 1994.

[13] K. R. Davidson. $C^{*}$-Algebras by Example. Fields Institute Monographs, 6. Amer. Math. Soc., Providence, RI, 1996.

[14] A. del Junco. Disjointness of measure-preserving transformations, minimal self-joinings and category. In: Ergodic Theory and Dynamical Systems, I (College Park, Md., 1979-80), 81-89. Prog. Math., 10, Birkhäuser, Boston, 1981.

[15] J. Dixmier. Les $C^{*}$-algèbres et leurs représentations. Gauthier-Villars, Paris, 1996.

[16] D. E. Evans. Completely positive quasi-free maps on the CAR algebra. Comm. Math. Phys. 70 (1979), 53-68.

[17] M. Foreman and B. Weiss. An anti-classification theorem for ergodic measure-preserving transformations. J. Eur. Math. Soc. 6 (2004), 277-292.

[18] T. Giordano, I. F. Putnam, and C. F. Skau. Topological orbit equivalence and $C^{*}$-crossed products. J. Reine Angew. Math. 469 (1995), 51-111.

[19] E. Glasner. Ergodic Theory via Joinings. American Mathematical Society, Providence, R.I., 2003.

[20] E. Glasner and J. L. King. A zero-one law for dynamical properties. In: Topological Dynamics and Applications (Minneapolis, MN, 1995), 231-242. Contemp. Math., 215, Amer. Math. Soc., Providence, RI, 1998.

[21] U. Haagerup. The standard form of von Neumann algebras. Math. Scand. 37 (1975), 271-283.

[22] U. Haagerup and C. Winsløw. The Effros-Maréchal topology in the space of von Neumann algebras. Amer. J. Math. 120 (1998), 567-617.

[23] P. R. Halmos and J. von Neumann. Operator methods in classical mechanics, II. Ann. of Math. (2) 43 (1942), 332-350.

[24] L. A. Harrington, A. S. Kechris, and A. Louveau. A Glimm-Effros dichotomy for Borel equivalence relations. J. Amer. Math. Soc. 3 (1990), 903-928.

[25] G. Hjorth. Non-smooth infinite-dimensional group representations. Preprint, 1997.

[26] G. Hjorth. Classification and Orbit Equivalence Relations. American Mathematical Society, Providence, RI, 2000.

[27] G. Hjorth. On invariants for measure preserving transformations. Fund. Math. 169 (2001), 51-84.

[28] V. F. R. Jones. Actions of finite groups on the hyperfinite $I I_{1}$ factor. Mem. Amer. Math. Soc. 237 (1980).

[29] R. Kallman. A generalization of free action. Duke Math. J. 36 (1969), 781-789.

[30] Y. Kawahigashi. Cohomology of actions of discrete groups on factors of type $\mathrm{II}_{1}$. Pac. J. Math. 149 (1991), 303-317.

[31] A. S. Kechris. Global aspects of ergodic group actions and equivalence relations. Preprint, 2007.

[32] A. S. Kechris and N. E. Sofronidis. A strong generic ergodicity property of unitary and self-adjoint operators. Ergod. Th. Dynam. Sys. 21 (2001), 1459-1479.

[33] D. Kerr and H. Li. Independence in topological and $C^{*}$-dynamics. Math. Ann. 338 (2007), 869-926.

[34] D. Kerr and H. Li. Combinatorial independence in measurable dynamics. Preprint, 2007.

[35] D. Kerr and M. Pichot. Asymptotic Abelianness, weak mixing, and property T. To appear in J. reine angew. Math.

[36] E. Lindenstrauss and B. Weiss. Mean topological dimension. Israel J. Math. 115 (2000), 1-24.

[37] S. Neshveyev and E. Størmer. Dynamical Entropy in Operator Algebras. Ergebnisse der Mathematik und ihrer Grenzgebiete, 3. Folge, vol. 50. Springer, Berlin, 2006.

[38] A. Ocneanu. Actions of discrete amenable groups on von Neumann algebras. Lecture Notes in Math., 1138. Springer-Verlag, Berlin, 1985.

[39] D. S. Ornstein. Some new results in the Kolmogorov-Sinai theory of entropy and ergodic theory. Bull. Amer. Math. Soc. 77 (1971), 878-890.

[40] D. S. Ornstein. Ergodic theory, Randomness, and Dynamical Systems. Yale Mathematical Monographs, 5. Yale University Press, New Haven, Conn., 1974. 
[41] G. K. Pedersen. $C^{*}$-algebras and their Automorphism Groups. Academic Press Inc., London, 1979.

[42] S. Popa. Singular maximal abelian *-subalgebras in continuous von Neumann algebras. J. Funct. Anal. 50 (1983), 151-166.

[43] S. Popa. Correspondences. INCREST preprint, 1986.

[44] M. A. Rieffel. Square-integrable representations of Hilbert algebras. J. Funct. Anal. 3 (1969), 265300.

[45] V. A. Rokhlin and A. A. Gurevič. Approximation theorems for measurable flows. (Russian) Izvestiya Akad. Nauk SSSR Ser. Mat. 14 (1950), 537-548.

[46] M. Takesaki. Theory of Operator Algebras III. Encyclopaedia of Mathematical Sciences, 127. Springer-Verlag, Berlin, 2003.

[47] S. Vaes. Rigidity results for Bernoulli actions and their von Neumann algebras (after Sorin Popa). Séminaire Bourbaki, exposé 961. Astérisque 311 (2007), 237-294.

[48] D. Voiculescu. A non-commutative Weyl-von Neumann theorem. Rev. Roum. Math. Pures et Appl. 21 (1976), 97-113.

[49] S. P. Wang. On isolated points in the dual spaces of locally compact groups. Math. Ann. 218 (1975), 19-34.

David Kerr, Department of Mathematics, Texas A\&M University, College Station TX 778433368, U.S.A.

E-mail address: kerr@math.tamu.edu

Hanfeng Li, Department of Mathematics, SUnY at Buffalo, Buffalo Ny 14260-2900, U.S.A. E-mail address: hfli@math.buffalo.edu

Mikaël Pichot, Department of Mathematical Sciences, University of Tokyo, 3-8-1 Komaba, TOKYO 153-8914, JAPAN

E-mail address: pichot@ms.u-tokyo.ac.jp 